\title{
AVERAGE BOX DIMENSIONS OF TYPICAL COMPACT SETS
}

\author{
Lars Olsen
}

University of St. Andrews, Department of Mathematics

St. Andrews, Fife KY16 9SS, Scotland; lo@st-and.ac.uk

\begin{abstract}
Let $X$ be a metric space and write $\mathcal{K}(X)$ for the family of non-empty compact subsets of $X$ equipped with the Hausdorff metric. The lower and upper box dimensions, denoted by $\underline{\operatorname{dim}}_{B}(E)$ and $\overline{\operatorname{dim}}_{B}(E)$, of a subset $E$ of $X$ are defined by

$$
\underline{\operatorname{dim}}_{B}(E)=\liminf _{r \searrow 0} \frac{\log N_{r}(E)}{-\log r}, \quad \overline{\operatorname{dim}}_{B}(E)=\limsup _{r \searrow 0} \frac{\log N_{r}(E)}{-\log r},
$$

where $N_{r}(E)$ is the smallest number of closed balls with centres in $E$ and radii equal to $r$ that are needed to cover $E$. In the 1980's, Gruber proved that the box counting function

$$
\frac{\log N_{r}(C)}{-\log r}
$$

of a typical compact set $C \in \mathcal{K}(X)$ diverges in the worst possible way as $r \searrow 0$. For example, Gruber proved that $\underline{\operatorname{dim}}_{B}(C)=0$ and $\overline{\operatorname{dim}}_{B}(C)=N$ for a typical $C \in \mathcal{K}\left(\mathbf{R}^{N}\right)$.

In this paper we prove that the box counting function $(*)$ of a typical compact set $C \in \mathcal{K}(X)$ is spectacularly more irregular than suggested by Gruber's result. In particular, we show the following surprising result: not only is the box counting function $(*)$ of a typical compact set $C \in \mathcal{K}(X)$ divergent as $r \searrow 0$, but it is so irregular that it remains spectacularly divergent as $r \searrow 0$ even after being "averaged" or "smoothened out" using powerful averaging methods including, for example, all higher order Hölder and Cesaro averages. As an application of our results we obtain strengthened versions of Gruber's result.
\end{abstract}

\section{Introduction}

Recall that a subset $E$ of a metric space $M$ is called co-meagre if its complement is meagre, and we say that a typical element $x \in M$ has property $P$ if the set $E=\{x \in M \mid x$ has property $P\}$ is co-meagre, see Oxtoby [Ox] for more details.

Let $(X, d)$ be a metric space and write $\mathcal{K}(X)$ for the set of non-empty compact subsets of $X$, i.e.

$$
\mathcal{K}(X)=\{C \subseteq X \mid C \text { is compact and non-empty }\} .
$$

We will always equip $\mathcal{K}(X)$ with the Hausdorff metric $d_{H}$. It is well-known that if $X$ is complete, then $\mathcal{K}(X)$ is a complete metric space when equipped with the Hausdorff metric.

The lower and upper box dimensions of a subset $E$ of $X$, denoted by $\underline{\operatorname{dim}}_{B}(E)$ and $\overline{\operatorname{dim}}_{B}(E)$, are defined by

$$
\underline{\operatorname{dim}}_{B}(E)=\liminf _{r \searrow 0} \frac{\log N_{r}(E)}{-\log r}, \quad \overline{\operatorname{dim}}_{B}(E)=\limsup _{r \searrow 0} \frac{\log N_{r}(E)}{-\log r},
$$

https://doi.org/10.5186/aasfm.2019.4406

2010 Mathematics Subject Classification: Primary 28A78, 28A80.

Key words: Box dimension, compact set, Hölder mean, Cesaro mean, Baire category. 
where

$$
\begin{aligned}
N_{r}(E)=\inf \{|\mathcal{B}| \mid & \mathcal{B} \text { is a family of closed balls } \\
& \text { with centres in } E \text { and radii equal to } r \text { that covers } E\} .
\end{aligned}
$$

If $\operatorname{dim}_{B}(E)=\overline{\operatorname{dim}}_{B}(E)$, then we say that the box dimension of $E$ exists and we denote the common value of $\underline{\operatorname{dim}}_{B}(E)$ and $\overline{\operatorname{dim}}_{B}(E)$ by $\operatorname{dim}_{B}(E)$. The reader is referred to $[\mathrm{Fa}]$ for an excellent and detailed discussion of box dimensions.

The purpose of this paper is to investigate the box dimensions, and, in particular, certain average box dimensions, of a typical element of $\mathcal{K}(X)$, i.e. of a typical compact subset of $X$. Box dimensions of typical compact sets have been investigated earlier. Indeed, in 1989, Gruber [Gr] proved the following result.

Theorem A. [Gr] Let $X$ be a metric space. For $s \geq 0$, write

$$
\mathcal{K}_{s}(X)=\left\{C \in \mathcal{K}(X) \mid \underline{\operatorname{dim}}_{B}(C) \geq s\right\},
$$

and put

$$
\Delta(X)=\sup \left\{s \geq 0 \mid \overline{\mathcal{K}_{s}(X)}=\mathcal{K}(X)\right\} .
$$

For a typical compact set $C \in \mathcal{K}(X)$, we have

$$
\underline{\operatorname{dim}}_{B}(C)=0, \quad \overline{\operatorname{dim}}_{B}(C) \geq \Delta(X) .
$$

In particular, by putting $X=\mathbf{R}^{N}$, we obtain the following corollary from Theorem A.

Corollary B. [Gr] For a typical compact set $C \in \mathcal{K}\left(\mathbf{R}^{N}\right)$, we have

$$
\underline{\operatorname{dim}}_{B}(C)=0, \quad \overline{\operatorname{dim}}_{B}(C)=N .
$$

In this paper we investigate the box dimensions of a typical compact subsets further. In particular, we study the following two problems.

(1) Average dimensions. Theorem A and Corollary B exhibit the same dichotomy, namely, the lower box dimension of a typical compact set is as small as possible and the upper box dimension of a typical compact set is (in many cases) as big as possible. Other studies [MyRu,Ro] of typical compact sets show the same dichotomy. For example, $[\mathrm{Ro}]$ proves that a typical compact metric space (belonging to the family of all compact metric spaces equipped with the Gromov-Hausdorff metric) has lower box dimension equal to 0 and upper box dimension equal to $\infty$, and [MyRu] improves the lower bound $\Delta(X)$ in Theorem $\mathrm{A}$ for the upper box dimension of a typical compact subset of a metric space $X$. The purpose of this paper is to analyse this intriguing dichotomy in more detail. In order to do so, we introduce the following notation. Namely, for a subset $E$ of a metric space $X$, we define the box counting function $f_{E}:(0, \infty) \rightarrow[0, \infty]$ of $E$ by

$$
f_{E}(t)=\frac{\log N_{e^{-t}}(E)}{-\log e^{-t}}=\frac{\log N_{e^{-t}}(E)}{t} ;
$$

we note the function $N_{r}(E)$ is often referred to as the box counting function of $E$ however, the function $f_{E}(t)$ is more fundamental for this work, and we will therefore refer to this function as the box counting function of $E$. Using this notation, the box dimensions of $E$ are now given by

$$
\underline{\operatorname{dim}}_{B}(E)=\liminf _{t \rightarrow \infty} f_{E}(t), \quad \overline{\operatorname{dim}}_{B}(E)=\limsup _{t \rightarrow \infty} f_{E}(t)
$$


and Theorem A therefore shows that (in many cases) the box counting function $f_{C}(t)$ of a typical compact set $C \in \mathcal{K}(X)$ diverges in the worst possible way as $t \rightarrow \infty$. In this paper we will prove that the behaviour of the box counting function $f_{C}(t)$ of a typical compact set $C \in \mathcal{K}(X)$ is spectacularly more irregular than suggested by this result and the analogous results in [MyRu,Ro]. Namely, there are standard techniques, known as averaging systems, that (at least in some cases) can assign limiting values to divergent functions (the precise definitions will be given below), and the purpose of this paper is to show the following surprising result: not only is the box counting function $f_{C}(t)$ of a typical compact set $C$ divergent as $t \rightarrow \infty$, but it is so irregular that it remains spectacularly divergent as $t \rightarrow \infty$ even after being "averaged" or "smoothened out" using powerful averaging systems including, for example, all higher order Hölder and Cesaro averages. Indeed, if $X=\mathbf{R}^{N}$, then we show that for a typical compact set $C \in \mathcal{K}\left(\mathbf{R}^{n}\right)$, all higher order lower Hölder averages of the box counting function $f_{C}(t)$ are as small as possible, namely, equal to 0 , and all higher order upper Hölder averages of the box counting function $f_{C}(t)$ are as big as possible, namely, equal to $N$. This is the statement of the special case of our main results presented below.

Theorem 1.1. Special case of Theorem 2.2 and Theorem 3.1. For a compact subset $C$ of $\mathbf{R}^{N}$, we define the $n$ 'th order Hölder averages, denoted by $H_{n}(C ; t)$, of the box counting function $f_{C}(t)$ of $C$ inductively by

$$
H_{0}(C ; t)=f_{C}(t), \quad H_{n}(C ; t)=\frac{1}{t} \int_{1}^{t} H_{n-1}(C ; s) d s,
$$

for $n \in \mathbf{N}$, and we define the lower and upper n'th order Hölder average box dimensions of $C$ by

$$
\underline{\operatorname{dim}}_{B, n}^{H}(C)=\liminf _{t \rightarrow \infty} H_{n}(C ; t), \quad \overline{\operatorname{dim}}_{B, n}^{H}(C)=\limsup _{t \rightarrow \infty} H_{n}(C ; t) .
$$

A typical compact set $C \in \mathcal{K}\left(\mathbf{R}^{N}\right)$ now satisfies

$$
\underline{\operatorname{dim}}_{B, n}^{H}(C)=0, \quad \overline{\operatorname{dim}}_{B, n}^{H}(C)=N
$$

for all $n \in \mathbf{N} \cup\{0\}$.

We emphasise that Theorem 1.1 is merely a special case of the more general results presented in Theorem 2.2 and Theorem 3.1. It is instructive to compare the statement in Theorem 1.1 with Gruber's Corollary B. While Corollary B shows the box counting function $f_{C}(t)$ of a typical $C \in \mathcal{K}\left(\mathbf{R}^{N}\right)$ diverges in the worst possible way, Theorem 1.1 shows that all higher order Hölder averages of $f_{C}(t)$ also diverge in the worst possible way.

(2) Local dimensions. In general, the lower bound $\Delta(X)$ for the upper box dimension $\overline{\operatorname{dim}}_{B}(C)$ of a typical compact set $C \in \mathcal{K}(X)$ in Theorem A is not sharp, i.e., in general, it is not true that $\overline{\operatorname{dim}}_{B}(C)=\Delta(X)$ for a typical compact set $C \in$ $\mathcal{K}(X)$. Indeed, in general, it is not even true that there is a positive number $D(X)$ such that $\overline{\operatorname{dim}}_{B}(C)=D(X)$ for a typical compact set $C \in \mathcal{K}(X)$. For example, if we let $Q=[0,1]^{2}$ denote the closed unit cube and $I=[2,3] \times\{0\}$, and put

$$
X=Q \cup I,
$$


then $\mathcal{K}(Q)$ and $\mathcal{K}(I)$ are non-empty and open subsets of $\mathcal{K}(X)$, and it follows easily from Theorem A that

$$
\overline{\operatorname{dim}}_{B}(C)= \begin{cases}2 & \text { for a typical compact set } C \in \mathcal{K}(Q) ; \\ 1 & \text { for a typical compact set } C \in \mathcal{K}(I) .\end{cases}
$$

In particular, this shows that there is no number $D(X)$ such that $\overline{\operatorname{dim}}_{B}(C)=D(X)$ for a typical compact set $C \in \mathcal{K}(X)$. Instead, we show that there is a "local dimension function" $\Delta(X ; \cdot): X \rightarrow \mathbf{R}$ such that if $X$ is an arbitrary separable metric space, then

$$
\overline{\operatorname{dim}}_{B}(C) \geq \sup _{x \in X} \Delta(X ; x)
$$

for a typical compact set $C \in \mathcal{K}(X)$, see Corollary 2.6. More importantly, we also show that for a large class of separable metric spaces $X$, the lower bound $\sup _{x \in X} \Delta(X ; x)$ for the upper box dimension $\overline{\operatorname{dim}}_{B}(C)$ of a typical compact set $C \in \mathcal{K}(X)$ is the exact value of $\overline{\operatorname{dim}}_{B}(C)$, i.e. we show that for a large class of separable metric spaces $X$ we have

$$
\overline{\operatorname{dim}}_{B}(C)=\sup _{x \in X} \Delta(X ; x)
$$

for a typical compact set $C \in \mathcal{K}(X)$, see Corollary 2.7. In fact, we prove more a general version of this result involving average box dimensions defined using arbitrary averaging systems, see Theorem 2.2 and Corollary 2.4.

\section{Statements of results}

2.1. Average dimension. We start by recalling the definition of an averaging (or summability) system; the reader is referred to Hardy's classical text [Ha] for a systematic treatment of averaging systems.

Definition. Averaging system. An averaging system is a family $\Pi=\left(\Pi_{t}\right)_{t \geq t_{0}}$ with $t_{0}>0$ such that:

(i) $\Pi_{t}$ is a finite Borel measure on $\left[t_{0}, \infty\right)$;

(ii) $\Pi_{t}$ has compact support;

(iii) The Consistency Condition: If $f:\left[t_{0}, \infty\right) \rightarrow[0, \infty)$ is a positive measurable function and there is a real number $a$ such that $f(t) \rightarrow a$ as $t \rightarrow \infty$, then $\int f d \Pi_{t} \rightarrow a$ as $t \rightarrow \infty$.

If $f:\left[t_{0}, \infty\right) \rightarrow[0, \infty)$ is a positive measurable function, then we define lower and upper П-average of $f$ by

$$
\underline{A}_{\Pi} f=\liminf _{t \rightarrow \infty} \int f d \Pi_{t}
$$

and

$$
\bar{A}_{\Pi} f=\limsup _{t \rightarrow \infty} \int f d \Pi_{t}
$$

respectively.

Applying averaging systems to the box counting function $f_{E}(t)$ in (1.1) leads to our key definition, namely, the definition of average box dimensions.

Definition. Average box dimension. Let $X$ a metric space and let $\Pi=\left(\Pi_{t}\right)_{t \geq t_{0}}$ be an averaging system. For a subset $E$ of $X$, we define the lower and upper $\Pi$-average 
box dimensions of $E$ by

$$
\underline{\operatorname{dim}}_{\Pi, B}(E)=\underline{A}_{\Pi} f_{E}=\liminf _{t \rightarrow \infty} \int \frac{\log N_{e^{-s}}(E)}{s} d \Pi_{t}(s)
$$

and

respectively.

$$
\overline{\operatorname{dim}}_{\Pi, B}(E)=\bar{A}_{\Pi} f_{E}=\limsup _{t \rightarrow \infty} \int \frac{\log N_{e^{-s}}(E)}{s} d \Pi_{t}(s),
$$

We note that box dimensions are, in fact, average box dimensions. Indeed, if $X$ a metric space and we let $\Pi$ denote the average system defined by $\Pi=\left(\delta_{t}\right)_{t \geq 1}$ (where $\delta_{t}$ denotes the Dirac measure concentrated at $t$ ), then clearly

$$
\underline{\operatorname{dim}}_{\Pi, B}(E)=\underline{\operatorname{dim}}_{B}(E), \quad \overline{\operatorname{dim}}_{\Pi, B}(E)=\overline{\operatorname{dim}}_{B}(E)
$$

for all subsets $E$ of $X$. Below we list some basic properties (and lack of properties) of average box dimensions.

Proposition 2.1. Basic properties of average dimensions. Let $X$ a metric space and let $\Pi=\left(\Pi_{t}\right)_{t \geq t_{0}}$ be an averaging system.

(1) $\underline{\operatorname{dim}}_{\Pi, B}$ and $\overline{\operatorname{dim}}_{\Pi, B}$ are monotone.

(2) $\underline{\operatorname{dim}}_{\Pi, B}$ and $\overline{\operatorname{dim}}_{\Pi, B}$ are finitely sub-stable.

(3) $\underline{\operatorname{dim}}_{\Pi, B}$ and $\overline{\operatorname{dim}}_{\Pi, B}$ are, in general, not finitely stable.

Recall, that if $D: \mathcal{K}(X) \rightarrow \mathbf{R}$ is a function, then $D$ is called monotone if $D(A) \leq D(B)$ for all $A, B \in \mathcal{K}(X)$ with $A \subseteq B ; D$ is called finitely sub-stable if $\max (D(A), D(B)) \leq D(A \cup B)$ for all $A, B \in \mathcal{K}(X)$; and $D$ is called finitely stable if $\max (D(A), D(B))=D(A \cup B)$ for all $A, B \in \mathcal{K}(X)$.

Proof. (1)-(2) These statements are clear.

(3) It is well-known that $\underline{\operatorname{dim}}_{B}$ (and hence, in particular, $\underline{\operatorname{dim}}_{\Pi, B}$ ) is not, in general, finitely stable, see, for example, [Fa]. Finally, in Section 4 we present an example showing that $\overline{\operatorname{dim}}_{\Pi, B}$ is not, in general, finitely stable.

Note that while $\overline{\operatorname{dim}}_{\Pi, B}$, in general, is not finitely stable, it is nevertheless true (and well-known, see, for example [Fa]) that $\overline{\operatorname{dim}}_{B}$ is finitely stable.

\subsection{Average box dimensions of typical compact sets: The main result.} We now state the main result in the paper, namely, Theorem 2.2 below. This result shows that the behaviour of the box counting function $f_{C}(t)$ of a typical set compact $C \in \mathcal{K}(X)$ is so irregular that remains divergent as $t \rightarrow \infty$ even after being "averaged" using arbitrary averaging systems.

Theorem 2.2. Let $X$ a metric space and let $\Pi=\left(\Pi_{t}\right)_{t \geq t_{0}}$ be an averaging system. For $x \in X$ and $r, s>0$, write

$$
\begin{aligned}
\mathcal{K}(X ; x, r) & =\{C \in \mathcal{K}(C) \mid C \subseteq \overline{B(x, r)}\}, \\
\mathcal{K}_{\Pi, s}(X ; x, r) & =\left\{C \in \mathcal{K}(C) \mid C \subseteq \overline{B(x, r)}, \overline{\left.\operatorname{dim}_{\Pi, B}(C) \geq s\right\},}\right.
\end{aligned}
$$

and let

$$
\begin{aligned}
\Delta_{\Pi}(X ; x, r) & =\sup \left\{s \geq 0 \mid \overline{\mathcal{K}_{\Pi, s}(X ; x, r)}=\mathcal{K}(X ; x, r)\right\}, \\
\Delta_{\Pi}(X ; x) & =\sup _{r>0} \Delta_{\Pi}(X ; x, r) .
\end{aligned}
$$


(1) For a typical $C \in \mathcal{K}(X)$, we have

$$
\underline{\operatorname{dim}}_{\Pi, B}(C)=0 .
$$

(2) If, in addition, $X$ is separable, then for a typical $C \in \mathcal{K}(X)$, we have

$$
\overline{\operatorname{dim}}_{\Pi, B}(C) \geq \sup _{x \in C} \Delta_{\Pi}(X ; x) .
$$

The proof of Theorem 2.2 is given in Sections 5-7. Section 5 contains various technical auxiliary results. The proof of Theorem 2.2.(1) is given in Section 6 and the proof of Theorem 2.2.(2) is given in Section 7.

Below we present several corollaries and applications of Theorem 2.2. In particular, we consider the following two applications of Theorem 2.2. In Section 2.3 we study several special cases of Theorem 2.2 where the lower bound for the upper average dimension of a typical compact set provides the exact value and/or can be computed explicitly, and in Section 2.4 we apply Theorem 2.2 to the average system $\Pi=\left(\delta_{t}\right)_{t \geq 1}$ leading to strengthened versions of Gruber's Theorem A.

2.3. Average box dimensions of typical compact sets: Some corollaries. We will now show that the lower bound for the upper average box dimension of a typical compact set in Theorem 2.2 is sharp for a certain class of metric spaces. In order to define this class of metric spaces, we introduce the following terminology.

Definition. $\Pi$-homogenous. Let $\Pi=\left(\Pi_{t}\right)_{t \geq t_{0}}$ be an averaging system. A metric spaces $X$ is called $\Pi$-homogenous if $\overline{\operatorname{dim}}_{\Pi, B}(G)=\overline{\operatorname{dim}}_{\Pi, B}(X)$ for every non-empty open subset $G$ of $X$.

Before stating our next result, we first note that many natural spaces are Пhomogenous.

Proposition 2.3. Let $\Pi$ be an averaging system.

(1) If $X \subseteq \mathbf{R}^{N}$ is quasi-self-similar from above, then $X$ is $\Pi$-homogenous; recall, that a metric space $(X, d)$ is called quasi-self-similar from above if there are constants $R, m>0$ such that for all $x \in X$ and all $0<r<R$ there is a function $\varphi: X \rightarrow B(x, r)$ such that if $\rho>0$ and $z \in X$, then there is $\zeta \in B(x, r)$ with $B\left(\zeta, m^{-1} \rho r\right) \subseteq \varphi(B(z, \rho)) \subseteq B(\zeta, m \rho r)$, see [O'Ne, p. 238; HaYa, Definition 1, p. 289].

(2) If $X \subseteq \mathbf{R}^{N}$ is self-conformal and satisfies the Open Set Condition, then $X$ is $\Pi$-homogenous.

(3) If $X \subseteq \mathbf{R}^{N}$ satisfies $X \subseteq X^{\circ-}$, then $X$ is $\Pi$-homogenous.

Proof. (1) Let $B$ be a ball in $X$. Since $X$ is quasi-self-similar from above there is a bi-Lipschitz function $f$ from $X$ into $B$. Since $f$ is bi-Lipschitz, it is not difficult to show that there is a constant $c>1$ such that

$$
N_{r}(X) \leq c N_{\frac{1}{c} r}(f(X))
$$

for all $r>0$. Also, since $f(X)$ is a subset of Euclidean space $\mathbf{R}^{N}$, it is not difficult to see that there is a further constant $k>1$ such that

$$
N_{r}(f(X)) \leq k N_{c r}(f(X))
$$

for all $r>0$. Combining (2.2) and (2.3), we conclude that

$$
N_{r}(X) \leq c k N_{r}(f(X))
$$


for all $r>0$. This clearly implies that $\overline{\operatorname{dim}}_{\Pi, B}(X) \leq \overline{\operatorname{dim}}_{\Pi, B}(f(X))$, and using the fact that $B \subseteq X$ and $f(X) \subseteq B$, we therefore conclude that $\overline{\operatorname{dim}}_{\Pi, B}(X)=\overline{\operatorname{dim}}_{\Pi, B}(B)$.

(2)-(3) These statement follow immediately from (1).

The next result says that if $X$ is a finite union of $\Pi$-homogenous spaces, then the lower bound for the upper average box dimension of a typical compact set in Theorem 2.2 is the exact value.

Corollary 2.4. Let $(X, d)$ be a metric space with $X=\bigcup_{i=1}^{n} X_{i}$ where inf $x_{x \in X_{i}, y \in X_{j}}$ $d(x, y)>0$ for all $i, j$ with $i \neq j$, and let $\Pi=\left(\Pi_{t}\right)_{t \geq t_{0}}$ be an averaging system. Assume that the following three conditions are satisfied:

(i) $X_{i}$ is $\Pi$-homogenous for all $i$;

(ii) Closed and bounded subsets of $X$ are compact;

(iii) If $n>1$, then assume, in addition, that $\overline{\operatorname{dim}}_{\Pi, B}$ is finitely stable.

Then the following statements hold.

(1) For all $C \in \mathcal{K}(X)$, we have

$$
0 \leq \underline{\operatorname{dim}}_{\Pi, B}(C) \leq \overline{\operatorname{dim}}_{\Pi, B}(C) \leq \sup _{C \cap X_{i} \neq \varnothing} \overline{\operatorname{dim}}_{\Pi, B}\left(X_{i}\right) .
$$

In particular, if the box dimension of $X_{i}$ exists for all $i$, then for all $C \in \mathcal{K}(X)$, we have

$$
0 \leq \underline{\operatorname{dim}}_{\Pi, B}(C) \leq \overline{\operatorname{dim}}_{\Pi, B}(C) \leq \sup _{C \cap X_{i} \neq \varnothing} \operatorname{dim}_{B}\left(X_{i}\right) .
$$

(2) If, in addition, $X$ is separable, then for a typical $C \in \mathcal{K}(X)$, we have

$$
\underline{\operatorname{dim}}_{\Pi, B}(C)=0, \quad \overline{\operatorname{dim}}_{\Pi, B}(C)=\sup _{C \cap X_{i} \neq \varnothing} \overline{\operatorname{dim}}_{\Pi, B}\left(X_{i}\right) .
$$

In particular, if $X$ is separable and the box dimension of $X_{i}$ exists for all $i$, then for a typical $C \in \mathcal{K}(X)$, we have

$$
\underline{\operatorname{dim}}_{\Pi, B}(C)=0, \quad \overline{\operatorname{dim}}_{\Pi, B}(C)=\sup _{C \cap X_{i} \neq \varnothing} \operatorname{dim}_{B}\left(X_{i}\right) .
$$

Proof. (1) It is clear that $0 \leq \underline{\operatorname{dim}}_{\Pi, B}(C) \leq \overline{\operatorname{dim}}_{\Pi, B}(C)$ and it follows from (iii) that $\overline{\operatorname{dim}}_{\Pi, B}(C)=\overline{\operatorname{dim}}_{\Pi, B}\left(\bigcup_{C \cap X_{i} \neq \varnothing} C \cap X_{i}\right)=\sup _{C \cap X_{i} \neq \varnothing} \overline{\operatorname{dim}}_{\Pi, B}\left(C \cap X_{i}\right) \leq$ $\sup _{C \cap X_{i} \neq \varnothing} \overline{\operatorname{dim}}_{\Pi, B}\left(X_{i}\right)$.

(2) It clearly suffices to show that if $C \in \mathcal{K}(X)$, then

$$
\sup _{C \cap X_{i} \neq \varnothing} \overline{\operatorname{dim}}_{\Pi, B}\left(X_{i}\right) \leq \sup _{x \in C} \Delta_{\Pi}(X ; x) .
$$

To prove (2.4) it clearly suffices to show that:

$$
\text { if } i=1, \ldots, n \text { and } x \in X_{i} \text {, then } \overline{\operatorname{dim}}_{\Pi, B}\left(X_{i}\right) \leq \Delta_{\Pi}(X ; x) .
$$

We will now prove (2.5). We therefore fix $i$ and $x \in X_{i}$. For brevity write $s=\overline{\operatorname{dim}}_{\Pi, B}\left(X_{i}\right)$, and note that in order to prove the inequality $s \leq \Delta_{\Pi}(X ; x)$, it suffices to show that:

$$
\text { there is } r>0 \text { such that } s \leq \Delta_{\Pi}(X ; x, r) \text {. }
$$

In order to prove prove (2.6), it is clearly sufficient to show that:

$$
\text { there is } r>0 \text { such that } \overline{\mathcal{K}_{\Pi, s}(X ; x, r)}=\mathcal{K}(X ; x, r) \text {. }
$$

We will now prove (2.7). First note that we may choose $0<r<\min _{i \neq j} \operatorname{dist}\left(X_{i}\right.$, $\left.X_{j}\right)$. We now claim that $\overline{\mathcal{K}_{\Pi, s}(X ; x, r)}=\mathcal{K}(X ; x, r)$. In order to prove this we fix a 
compact set $K$ with $K \subseteq \overline{B(x, r)}$ and $\delta>0$. We must now find $C \in \mathcal{K}_{\Pi, s}(X ; x, r)$ with $d_{H}(K, C)<\delta$. First choose a finite subset $E$ of $\overline{B(x, r)}$ such that $d_{H}(K, E)<\frac{\delta}{3}$. Next, fix $y \in E$ and note that since $y \in E \subseteq \overline{B(x, r)}$, there is a point $x_{y} \in B(x, r)$ with $d\left(y, x_{y}\right)<\frac{\delta}{3}$ where $d$ denotes the metric in $X$. Finally, since $x_{y} \in B(x, r)$, we can choose $r_{y}>0$ such that $B\left(x_{y}, 2 r_{y}\right) \subseteq B(x, r)$ and $2 r_{y}<\frac{\delta}{3}$. Now put $C=$ $\bigcup_{y \in E} \overline{B\left(x_{y}, r_{y}\right)}$, and note that it follows from Condition (ii) that $C$ is compact. Since $E$ is non-empty we can find $y_{0} \in E$. We now have $B\left(x_{y_{0}}, r_{y_{0}}\right) \subseteq B(x, r) \subseteq X_{i}$, and we therefore conclude from Condition (i) and the definition of $C$ that

$$
\begin{aligned}
C & =\bigcup_{y \in E} \overline{B\left(x_{y}, r_{y}\right)} \subseteq \bigcup_{y \in E} B\left(x_{y}, 2 r_{y}\right) \subseteq B(x, r) \subseteq \overline{B(x, r)}, \\
\overline{\operatorname{dim}}_{\Pi, B}(C) & \geq \overline{\operatorname{dim}}_{\Pi, B}\left(\bigcup_{y \in E} \overline{B\left(x_{y}, r_{y}\right)}\right) \geq \overline{\operatorname{dim}}_{\Pi, B}\left(B\left(x_{y_{0}}, r_{y_{0}}\right)\right)=\overline{\operatorname{dim}}_{\Pi, B}\left(X_{i}\right),
\end{aligned}
$$

whence $C \in \mathcal{K}_{\Pi, s}(X ; x, r)$. We also have

$$
\begin{aligned}
d_{H}(K, C) & \leq d_{H}(K, E)+d_{H}\left(E, \bigcup_{y \in E}\left\{x_{y}\right\}\right)+d_{H}\left(\bigcup_{y \in E}\left\{x_{y}\right\}, \bigcup_{y \in E} \overline{B\left(x_{y}, r_{y}\right)}\right) \\
& <\frac{\delta}{3}+\frac{\delta}{3}+\frac{\delta}{3}=\delta .
\end{aligned}
$$

This completes the proof of (2.7).

If $X$ is a subset of a Euclidean space, then the statement in Corollary 2.4 simplifies considerably; this is the statement of the next corollary.

Corollary 2.5. Let $X$ be a subset of $\mathbf{R}^{N}$ and let $\Pi=\left(\Pi_{t}\right)_{t \geq t_{0}}$ be an averaging system. Assume that $X$ is $\Pi$-homogenous. Then the following statements hold.

(1) For all $C \in \mathcal{K}(X)$, we have

$$
0 \leq \underline{\operatorname{dim}}_{\Pi, B}(C) \leq \overline{\operatorname{dim}}_{\Pi, B}(C) \leq \operatorname{dim}_{\Pi, B}(X) .
$$

In particular, if the box dimension of $X$ exists then for all $C \in \mathcal{K}(X)$, we have

$$
0 \leq \underline{\operatorname{dim}}_{\Pi, B}(C) \leq \overline{\operatorname{dim}}_{\Pi, B}(C) \leq \operatorname{dim}_{B}(X) .
$$

(2) For a typical $C \in \mathcal{K}(X)$, we have

$$
\underline{\operatorname{dim}}_{\Pi, B}(C)=0, \quad \overline{\operatorname{dim}}_{\Pi, B}(C)=\operatorname{dim}_{\Pi, B}(X) .
$$

In particular, if the box dimension of $X$ exists, then for a typical $C \in \mathcal{K}(X)$, we have

$$
\underline{\operatorname{dim}}_{\Pi, B}(C)=0, \quad \overline{\operatorname{dim}}_{\Pi, B}(C)=\operatorname{dim}_{B}(X) .
$$

Proof. This follows immediately from Corollary 2.4 .

2.4. Box dimensions of typical compact sets. Since the average dimensions associated with the average system $\Pi=\left(\delta_{t}\right)_{t \geq 1}$ equal the usual box dimensions (see (2.1)), applying Theorem 2.2 to the average system $\Pi=\left(\delta_{t}\right)_{t \geq 1}$ leads to a strengthened version of Gruber's result in Theorem A; this is the content of Corollary 2.6 below.

Corollary 2.6. Let $X$ a metric space For $x \in X$ and $r, s>0$, write

$$
\begin{aligned}
\mathcal{K}(X ; x, r) & =\{C \in \mathcal{K}(C) \mid C \subseteq \overline{B(x, r)}\}, \\
\mathcal{K}_{s}(X ; x, r) & =\left\{C \in \mathcal{K}(C) \mid C \subseteq \overline{B(x, r)}, \quad \overline{\operatorname{dim}}_{B}(C) \geq s\right\},
\end{aligned}
$$


and let

$$
\begin{aligned}
\Delta(X ; x, r) & =\sup \left\{s \geq 0 \mid \overline{\mathcal{K}_{s}(X ; x, r)}=\mathcal{K}(X ; x, r)\right\}, \\
\Delta(X ; x) & =\sup _{r>0} \Delta(X ; x, r) .
\end{aligned}
$$

For a typical $C \in \mathcal{K}(X)$, we have

$$
\underline{\operatorname{dim}}_{B}(C)=0 .
$$

If, in addition, $X$ is separable, then for a typical $C \in \mathcal{K}(X)$, we have

$$
\overline{\operatorname{dim}}_{B}(C) \geq \sup _{x \in C} \Delta(X ; x) .
$$

Proof. Let $\Pi$ denote the average system defined by $\Pi=\left(\delta_{t}\right)_{t \geq 1}$. Since $\underline{\operatorname{dim}}_{\Pi, B}(E)$ $=\underline{\operatorname{dim}}_{B}(E)$ and $\overline{\operatorname{dim}}_{\Pi, B}(E)=\overline{\operatorname{dim}}_{B}(E)$ for all subsets $E$ of $X$ (see (2.1)), the desired statement follows immediately from applying Theorem 2.2 to $\Pi=\left(\delta_{t}\right)_{t \geq 1}$.

Note that if $\Delta(X)$ denotes the number in Theorem $\mathrm{A}$ and $\Delta(X ; x)$ denotes the number in Corollary 2.6, then clearly

$$
\sup _{x \in C} \Delta(X ; x) \geq \Delta(X)
$$

for all compact subsets $C$ of $X$, and Corollary 2.6 is therefore a strengthening of Theorem A. It is also instructive to apply Corollary 2.4 to the average system $\Pi=$ $\left(\delta_{t}\right)_{t \geq 1}$. This leads to exact formulas for the box dimensions of typical compact subsets of finite unions of homogenous subsets of $\mathbf{R}^{N}$, and is the content of the next result.

Corollary 2.7. Let $(X, d)$ be a subset of $\mathbf{R}^{N}$ with $X=\bigcup_{i=1}^{n} X_{i}$ where $\inf _{x \in X_{i}, y \in X_{j}}$ $d(x, y)>0$ for all $i, j$ with $i \neq j$. Assume that $\overline{\operatorname{dim}}_{B}(G)=\overline{\operatorname{dim}}_{B}\left(X_{i}\right)$ for all $i$ and all non-empty open subsets $G$ of $X_{i}$. Then the following statements hold.

(1) For all $C \in \mathcal{K}(X)$, we have

$$
0 \leq \underline{\operatorname{dim}}_{B}(C) \leq \overline{\operatorname{dim}}_{B}(C) \leq \sup _{C \cap X_{i} \neq \varnothing} \overline{\operatorname{dim}}_{B}\left(X_{i}\right)
$$

(2) If, in addition, $X$ is separable, then for a typical $C \in \mathcal{K}(X)$, we have

$$
\underline{\operatorname{dim}}_{B}(C)=0, \quad \overline{\operatorname{dim}}_{B}(C)=\sup _{C \cap X_{i} \neq \varnothing} \overline{\operatorname{dim}}_{B}\left(X_{i}\right) .
$$

Proof. Let $\Pi$ denote the average system defined by $\Pi=\left(\delta_{t}\right)_{t \geq 1}$. Since $\underline{\operatorname{dim}}_{\Pi, B}(E)$ $=\underline{\operatorname{dim}}_{B}(E)$ and $\overline{\operatorname{dim}}_{\Pi, B}(E)=\overline{\operatorname{dim}}_{B}(E)$ for all subsets $E$ of $X$ (see (2.1)) and the upper box dimension is finitely stable (see $[\mathrm{Fa}]$ ), the desired statement follows immediately from applying Corollary 2.4 to $\Pi=\left(\delta_{t}\right)_{t \geq 1}$.

As above, we note that Corollary 2.7 is a strengthening of Gruber's Theorem A. Corollary 2.7 is, in fact, in many cases strictly stronger than Gruber's Theorem A. For example, if we let $Q=[0,1]^{2}$ and $I=[2,3] \times\{0\}$, and put $X=Q \cup I$, then it follows from Gruber's Theorem A that

$$
\overline{\operatorname{dim}}_{B}(C) \geq 1
$$

for a typical $C \in \mathcal{K}(X)$, whereas Corollary 2.7 provides the exact value for the upper box dimension $\overline{\operatorname{dim}}_{B}(C)$ of a typical compact set $C \in \mathcal{K}(X)$, namely, Corollary 2.7 shows that

$$
\overline{\operatorname{dim}}_{B}(C)= \begin{cases}2 & \text { if } C \cap Q \neq \varnothing \\ 1 & \text { if } C \cap Q=\varnothing\end{cases}
$$


for a typical $C \in \mathcal{K}(X)$.

In Sections 3-4, we present several applications of Theorem 2.2 to two classical averaging methods $\Pi$, namely, Hölder and Cesaro averages.

\section{Hölder and Cesaro averages of the box dimension of a typical compact set}

Two of the most commonly used averaging method are Hölder averages and Cesaro averages. We will now define these average methods and apply them to the box counting function $f_{C}(t)$ of a compact set $C$. We first recall the definitions of the Hölder and Cesaro averages. For $a>0$ and a measurable function $f:(a, \infty) \rightarrow$ $[0, \infty)$, we define $M f:(a, \infty) \rightarrow[0, \infty)$ by

$$
(M f)(t)=\frac{1}{t} \int_{a}^{t} f(s) d s
$$

For a positive integer $n$, we now define the lower and upper $n$ 'th order Hölder averages of $f$ by

$$
\underline{H}_{n} f=\liminf _{t \rightarrow \infty}\left(M^{n} f\right)(t), \quad \bar{H}_{n} f=\limsup _{t \rightarrow \infty}\left(M^{n} f\right)(t) .
$$

The Cesaro averages are defined as follows. First, we define If: $(a, \infty) \rightarrow[0, \infty)$ by

$$
(I f)(t)=\int_{a}^{t} f(s) d s
$$

For a positive integer $n$, we now define the lower and upper $n$ 'th order Cesaro averages of $f$ by

$$
\underline{C}_{n} f=\liminf _{t \rightarrow \infty} \frac{n !}{t^{n}}\left(I^{n} f\right)(t), \quad \bar{C}_{n} f=\limsup _{t \rightarrow \infty} \frac{n !}{t^{n}}\left(I^{n} f\right)(t) .
$$

It is well-known that the Hölder and Cesaro averages satisfy the following inequalities, namely,

$$
\begin{aligned}
& \liminf _{t \rightarrow \infty} f(t)=\underline{H}_{0} f \leq \underline{H}_{1} f \leq \underline{H}_{2} f \leq \ldots \leq \bar{H}_{2} f \leq \bar{H}_{1} f \leq \bar{H}_{0} f=\limsup _{t \rightarrow \infty} f(t), \\
& \liminf _{t \rightarrow \infty} f(t)=\underline{C}_{0} f \leq \underline{C}_{1} f \leq \underline{C}_{2} f \leq \ldots \leq \bar{C}_{2} f \leq \bar{C}_{1} f \leq \bar{C}_{0} f=\limsup _{t \rightarrow \infty} f(t) .
\end{aligned}
$$

It is also well-known that the Hölder and Cesaro averages are averaging methods in the sense of the definition in Section 2.1. Indeed, if we for a positive integer $n$, define the averaging method $\Pi_{n}^{H}=\left(\Pi_{n, t}^{H}\right)_{t \geq a}$ by

$$
\Pi_{n, t}^{H}(B)=\frac{1}{(n-1) ! t} \int_{[a, t] \cap B}(\log t-\log s)^{n-1} d s
$$

for Borel subsets $B$ of $[a, \infty)$, then

$$
\underline{H}_{n} f=\liminf _{t} \int f d \Pi_{n, t}^{H}, \quad \bar{H}_{n} f=\limsup _{t} \int f d \Pi_{n, t}^{H},
$$

see, for example, [Ja, p. 675]. Similarly, if we for a positive integer $n$, define the averaging method $\Pi_{n}^{C}=\left(\Pi_{n, t}^{C}\right)_{t \geq a}$ by

$$
\Pi_{n, t}^{C}(B)=\frac{n}{t^{n}} \int_{[a, x] \cap B}(t-s)^{n-1} d s
$$

then

$$
\underline{C}_{n} f=\liminf _{t} \int f d \Pi_{n, t}^{C}, \quad \bar{C}_{n} f=\limsup _{t} \int f d \Pi_{n, t}^{C},
$$


see, for example, [Ha, pp. 110-111].

Using Hölder and Cesaro averages we can now introduce average Hölder and Cesaro box dimensions by applying the definitions of the Hölder and Cesaro averages to the function $f_{E}(t)=\frac{\log N_{e^{-t}}(E)}{t}$. This is the content of the next definition.

Definition. Average Hölder and Cesaro box dimensions. Let $X$ be a metric space. For a subset $E$ of $X$, we define the lower and upper $n$ 'th order average Hölder box dimension of $E$, denoted by $\underline{\operatorname{dim}}_{B, n}^{H}(E)$ and $\overline{\operatorname{dim}}_{B, n}^{H}(E)$, as the lower and upper $n$ 'th order Hölder average of the function $f_{E}(t)$ for $t \geq 1$, i.e. we put

$$
\underline{\operatorname{dim}}_{B, n}^{H}(E)=\underline{H}_{n} f_{E}, \quad \overline{\operatorname{dim}}_{B, n}^{H}(E)=\bar{H}_{n} f_{E} .
$$

Similarly, we define the lower and upper $n$ 'th order average Cesaro box dimension of $E$, denoted by $\underline{\operatorname{dim}}_{B, n}^{C}(E)$ and $\overline{\operatorname{dim}}_{B, n}^{C}(E)$, by

$$
\underline{\operatorname{dim}}_{B, n}^{C}(E)=\underline{C}_{n} f_{E}, \quad \overline{\operatorname{dim}}_{B, n}^{C}(E)=\bar{C}_{n} f_{E} .
$$

The higher order average Hölder and Cesaro box dimensions form a double infinite hierarchy in (at least) countably infnite many levels, namely, we have (using (3.1))

$$
\begin{aligned}
\underline{\operatorname{dim}}_{B}(E) & =\underline{\operatorname{dim}}_{B, 0}^{H}(E) \leq \underline{\operatorname{dim}}_{B, 1}^{H}(E) \leq \ldots \\
& \leq \overline{\operatorname{dim}}_{B, 1}^{H}(E) \leq \overline{\operatorname{dim}}_{B, 0}^{H}(E)=\overline{\operatorname{dim}}_{B}(E), \\
\underline{\operatorname{dim}}_{B}(E) & =\underline{\operatorname{dim}}_{B, 0}^{C}(E) \leq \underline{\operatorname{dim}}_{B, 1}^{C}(E) \leq \ldots \\
& \leq \overline{\operatorname{dim}}_{B, 1}^{C}(E) \leq \overline{\operatorname{dim}}_{B, 0}^{C}(E)=\overline{\operatorname{dim}}_{B}(E) .
\end{aligned}
$$

As an application of Corollary 2.5, we will now show that if $X$ is a $\Pi$-homogenous subset of $\mathbf{R}^{N}$, then the behaviour of a typical compact set $C \in \mathcal{K}(X)$ is so irregular that not even the hierarchies in (3.2) formed by taking Hölder and Cesaro averages of all orders are sufficiently powerful to "smoothen out" the behaviour of the box counting function $f_{C}(t)$ as $t \rightarrow \infty$.

Theorem 3.1. Let $X$ be a subset of $\mathbf{R}^{N}$. Assume that $X$ is $\Pi_{n}^{H}$-homogenous and $\Pi_{n}^{C}$-homogenous for all $n \in \mathbf{N} \cup\{0\}$. Then a typical compact set $C \in \mathcal{K}(X)$ satisfies:

$\underline{\operatorname{dim}}_{B, n}^{H}(C)=0, \overline{\operatorname{dim}}_{B, n}^{H}(C)=\overline{\operatorname{dim}}_{B, n}^{H}(X), \underline{\operatorname{dim}}_{B, n}^{C}(C)=0, \overline{\operatorname{dim}}_{B, n}^{C}(C)=\overline{\operatorname{dim}}_{B, n}^{C}(X)$,

for all $n \in \mathbf{N} \cup\{0\}$. In particular, if, in addition, the box dimension of $X$ exists, then a typical compact set $C \in \mathcal{K}(X)$ satisfies:

$$
\underline{\operatorname{dim}}_{B, n}^{H}(C)=\underline{\operatorname{dim}}_{B, n}^{C}(C)=0, \quad \overline{\operatorname{dim}}_{B, n}^{H}(C)=\overline{\operatorname{dim}}_{B, n}^{C}(C)=\operatorname{dim}_{B}(X),
$$

for all $n \in \mathbf{N} \cup\{0\}$.

Proof. This statement follows immediately from Corollary 2.5.

\section{An example}

In order to illustrate the above definitions and concepts, we present and elaborate on a simple and concrete example from [AAOPRR] of a (compact) subset $X$ of $\mathbf{R}$ and compute its box dimensions and its 1 'st order Hölder average box dimensions. We 
construct the set $X$ as follows. For $i=0,1,2,3,4$, define the map $S_{i}:[0,1] \rightarrow[0,1]$ by $S_{i}(x)=\frac{1}{5} x+\frac{i}{5}$. Let $N_{1}=1$ and $N_{n}=2^{n-2}$ for $n \geq 2$, and write

$$
\Sigma_{n}= \begin{cases}\left\{i_{1} \ldots i_{N_{n}} \mid i_{j} \in\{0,4\} \text { for all } j\right\} & \text { if } n \text { is even; } \\ \left\{i_{1} \ldots i_{N_{n}} \mid i_{j} \in\{0,2,4\} \text { for all } j\right\} & \text { if } n \text { is odd }\end{cases}
$$

i.e. $\Sigma_{n}$ is the family of all finite strings $\mathbf{i}=i_{1} \ldots i_{N_{n}}$ of length $N_{n}$ with entries $i_{j}$ from $\{0,2,4\}$ if $n$ is odd, and with entries $i_{j}$ from $\{0,4\}$ if $n$ is even. For $\mathbf{i}=i_{1} \ldots i_{N_{n}} \in \Sigma_{n}$, we write $S_{\mathbf{i}}=S_{i_{1}} \circ \cdots \circ S_{i_{N_{n}}}$. The set $X$ is now defined by

$$
X=\bigcap_{n} \bigcup_{\mathbf{i}_{1} \in \Sigma_{1}, \ldots, \mathbf{i}_{n} \in \Sigma_{n}} S_{\mathbf{i}_{1}} \circ \cdots \circ S_{\mathbf{i}_{n}}([0,1]) .
$$

The box dimensions of $X$ and the 1'st order Hölder average box dimensions of $X$ are given by the following formulas from [AAOPRR].

Theorem 4.1. [AAOPRR] Let $X$ be given by (4.1) and write $a=\frac{\log 2}{\log 5}$ and $b=\frac{\log 3}{\log 5}$. Then we have

$$
\begin{aligned}
\underline{\operatorname{dim}}_{B}(X) & =\frac{2}{3} a+\frac{1}{3} b \approx 0.51465, \\
\underline{\operatorname{dim}}_{B, 1}^{H}(X) & =\frac{2^{\frac{2}{3}}}{3} a+\left(1-\frac{2^{\frac{2}{3}}}{3}\right) b \approx 0.54930, \\
\overline{\operatorname{dim}}_{B, 1}^{H}(X) & =\left(1-\frac{2^{\frac{2}{3}}}{3}\right) a+\frac{2^{\frac{2}{3}}}{3} b \approx 0.56398, \\
\overline{\operatorname{dim}}_{B}(X) & =\frac{1}{3} a+\frac{2}{3} b \approx 0.59863 .
\end{aligned}
$$

It is instructive to present some numerical calculations illustrating the oscillatory behaviour in the definitions of the dimensions $\underline{\operatorname{dim}}_{B}(X), \underline{\operatorname{dim}}_{B, 1}^{H}(X), \overline{\operatorname{dim}}_{B, 1}^{H}(X)$ and $\overline{\operatorname{dim}}_{B}(X)$. Write $r_{n}=5^{-n}$ and put $\pi_{n}=\frac{\log N_{r_{n}}(X)}{-\log r_{n}}$. Since $\frac{\log r_{n+1}}{\log r_{n}} \rightarrow 1$, it follows from $[\mathrm{Fa}]$ that

$$
\underline{\operatorname{dim}}_{B}(X)=\liminf _{n} \pi_{n}, \quad \overline{\operatorname{dim}}_{B}(X)=\limsup _{n} \pi_{n}
$$

and it follows from [AAOPRR] that

$$
\underline{\operatorname{dim}}_{B, 1}^{H}(X)=\liminf _{n} \frac{1}{n} \sum_{i=1}^{n} \pi_{i}, \quad \overline{\operatorname{dim}}_{B, 1}^{H}(X)=\limsup _{n} \frac{1}{n} \sum_{i=1}^{n} \pi_{i} .
$$

Below we sketch the graphs of the sequences $\left(\pi_{n}\right)_{n}$ and $\left(\frac{1}{n} \sum_{i=1}^{n} \pi_{i}\right)_{n}$ illustrating their oscillatory behaviour.

Finally, we show that $\overline{\operatorname{dim}}_{B, 1}^{H}$ is not finitely stable. Specifically, we show that there is a subset $Y$ of $\mathbf{R}$ such that

$$
\overline{\operatorname{dim}}_{B, 1}^{H}(X \cup Y)>\max \left(\overline{\operatorname{dim}}_{B, 1}^{H}(X), \overline{\operatorname{dim}}_{B, 1}^{H}(Y)\right)
$$

Indeed, let $Y$ be any subset of $[2,3]$ such that the box $\operatorname{dimension} \operatorname{dim}_{B}(Y)$ of $Y$ exists and equals $\overline{\operatorname{dim}}_{B, 1}^{H}(X)$. Next, write $\tau_{n}=\frac{\log N_{r_{n}}(Y)}{-\log r_{n}}$, and note that since $\operatorname{dist}(X, Y)=$ $1>0$, an argument very similar to the proof of (4.3) shows that $\overline{\operatorname{dim}}_{B, 1}^{H}(X \cup$ $Y)=\limsup _{n} \frac{1}{n} \sum_{i=1}^{n} \max \left(\pi_{i}, \tau_{i}\right)$. Finally, since $\tau_{n} \rightarrow \operatorname{dim}_{B}(Y)=\overline{\operatorname{dim}}_{B, 1}^{H}(X)=$ $\limsup \frac{1}{m} \sum_{i=1}^{m} \pi_{i}$ and $\operatorname{dim}_{B}(Y)=\overline{\operatorname{dim}}_{B, 1}^{H}(Y)$, it now follows from the identity $\overline{\operatorname{dim}}_{B, 1}^{H}(X \cup Y)=\lim \sup _{n} \frac{1}{n} \sum_{i=1}^{n} \max \left(\pi_{i}, \tau_{i}\right)$, using arguments similar to those in [AAOPRR], that (4.4) holds. This concludes the example. 

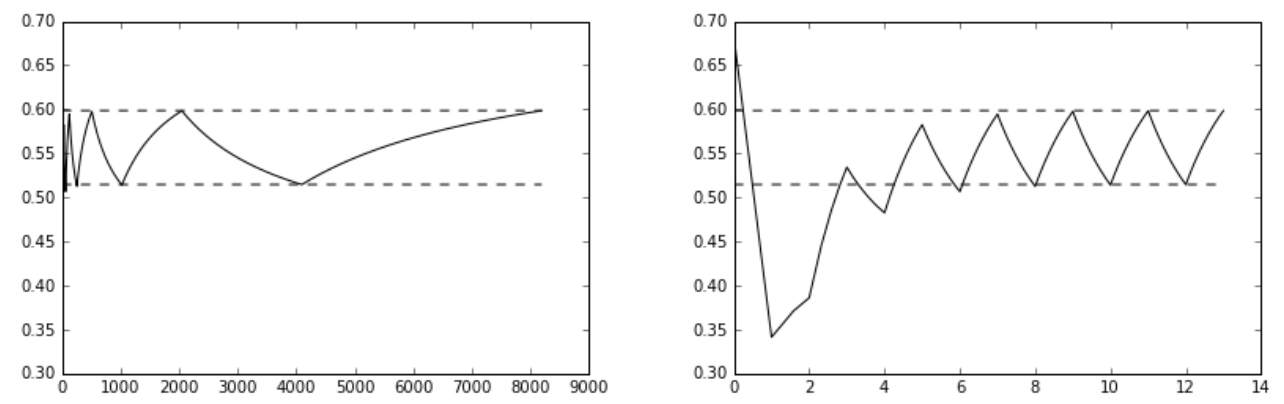

Figure 4.1. The figure on the left shows the points $\left(n, \pi_{n}\right)$ for $n \in\left\{1,2,3, \ldots, 2^{13}\right\}$, and the figure on the right shows the points $\left(\frac{\log n}{\log 2}, \pi_{n}\right)$ for $n \in\left\{1,2,3, \ldots, 2^{13}\right\}$. The two horizontal dashed lines intersect the vertical axis at $\underline{\operatorname{dim}}_{B}(X)=\liminf _{n} \pi_{n}=\frac{2}{3} a+\frac{1}{3} b \approx 0.51465$ and $\overline{\operatorname{dim}}_{B}(X)=$ $\lim \sup _{n} \pi_{n}=\frac{1}{3} a+\frac{2}{3} b \approx 0.59863$, respectively.
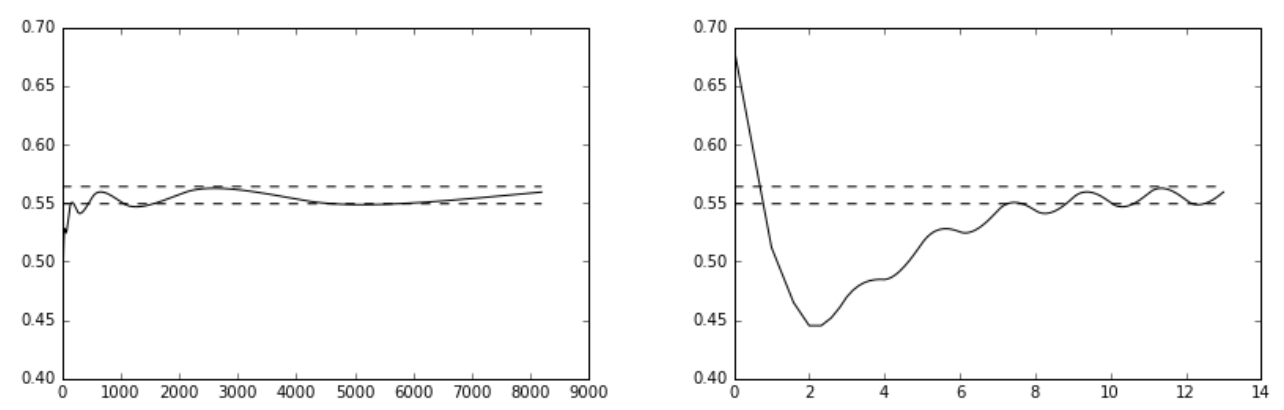

Figure 4.2. The figure on the left shows the points $\left(n, \frac{1}{n} \sum_{i=1}^{n} \pi_{i}\right)$ for $n \in\left\{1,2,3, \ldots, 2^{13}\right\}$, and the figure on the right shows the points $\left(\frac{\log n}{\log 2}, \frac{1}{n} \sum_{i=1}^{n} \pi_{i}\right)$ for $n \in\left\{1,2,3, \ldots, 2^{13}\right\}$. The two horizontal dashed lines intersect the vertical axis at $\underline{\operatorname{dim}}_{B, 1}^{H}(X)=\liminf \operatorname{li}_{n} \frac{1}{n} \sum_{i=1}^{n} \pi_{i}=\frac{2^{\frac{2}{3}}}{3} a+(1-$ $\left.\frac{2^{\frac{2}{3}}}{3}\right) b \approx 0.54930$ and $\overline{\operatorname{dim}}_{B, 1}^{H}(X)=\lim \sup _{n} \frac{1}{n} \sum_{i=1}^{n} \pi_{i}=\left(1-\frac{2^{\frac{2}{3}}}{3}\right) a+\frac{2^{\frac{2}{3}}}{3} b \approx 0.56398$, respectively.

\section{Proof of Theorem 2.2: Preliminary results}

In this section we collect some basic notation and a technical auxiliary lemma that will be used extensively in Sections 6-7. We first recall the definition of an $r$-covering set and the $r$-covering number $N_{r}(E)$ of a subset $E$ of a metric space. Below we will write $|\Lambda|$ for the cardinality of a set $\Lambda$.

Definition. $r$-covering set and $N_{r}(E)$. Let $(X, d)$ be a metric space. Fix $r>0$ and $E \subseteq X$. We say that a subset $\Lambda$ of $X$ is an $r$-covering subset of $E$ provided $\Lambda \subseteq E$ and or all $x \in E$, there is $y \in \Lambda$ such that $d(x, y) \leq r$. For $r>0$ and $E \subseteq X$, we define the $r$-covering number $N_{r}(E)$ of $E$ by

$$
N_{r}(E)=\inf \{|\Lambda| \mid \Lambda \text { is an } r \text {-covering subset of } E\} .
$$

Next, we define the dual notion, namely, we provide the definition of an $r$-packing set and the $r$-packing number $M_{r}(E)$ of a subset $E$ of a metric space.

Definition. $r$-separated set and $M_{r}(E)$. Let $(X, d)$ be a metric space. Fix $r>0$ and $E \subseteq X$. We say the a subset $\Lambda$ of $X$ is an $r$-separated subset of $E$ provided $\Lambda \subseteq E$ and for all $x, y \in \Lambda$ with $x \neq y$, we have $d(x, y) \geq r$. For $r>0$ and $E \subseteq X$, 
we define the $r$-packing number $M_{r}(E)$ of $E$ by

$$
M_{r}(E)=\sup \{|\Lambda| \mid \Lambda \text { is an } r \text {-separated subset of } E\} .
$$

The next results list some of the fundamental properties of the covering number $N_{r}(E)$ and the packing number $M_{r}(E)$; these properties will be used extensively in Sections 6-7.

Lemma 5.1. Let $X$ be a metric space and $r>0$.

(1) The function $N_{r}: \mathcal{K}(X) \rightarrow \mathbf{R}$ is lower semi-continuous.

(2) The function $M_{r}: \mathcal{K}(X) \rightarrow \mathbf{R}$ is upper semi-continuous.

(3) $N_{r}(E) \leq M_{r}(E)$ for all subsets $E$ of $X$.

Proof. This follows from [Gr, p. 152].

\section{Proof of the lower bound: Theorem 2.2.(1)}

The purpose of this section is to prove Theorem 2.2.(1). We first prove two auxiliary lemmas. The first lemma (i.e. Lemma 6.1) is standard and is a version of the reverse Fatou's lemma.

Lemma 6.1. The reverse Fatou's Lemma [St, Theorem 3.2.3]. Let $(M, \mathcal{E}, \mu)$ be a measure space and let $\left(\varphi_{n}\right)_{n}$ be a sequence of positive measurable functions $\varphi_{n}: M \rightarrow[0, \infty]$. If $\int \sup _{n} \varphi_{n} d \mu<\infty$, then $\lim \sup _{n} \int \varphi_{n} d \mu \leq \int \lim \sup _{n} \varphi_{n} d \mu$.

Lemma 6.2. Let $X$ be a metric space and let $\Pi=\left(\Pi_{t}\right)_{t \geq t_{0}}$ be an averaging system. Let $c \in \mathbf{R}$ and $t \geq t_{0}$. Then the set

$$
\left\{C \in \mathcal{K}(X) \mid \int \frac{\log M_{e^{-s}}(C)}{s} d \Pi_{t}(s)<c\right\}
$$

is open in $\mathcal{K}(X)$.

Proof. Write

$$
\begin{aligned}
F & =\mathcal{K}(X) \backslash\left\{C \in \mathcal{K}(X) \mid \int \frac{\log M_{e^{-s}}(C)}{s} d \Pi_{t}(s)<c\right\} \\
& =\left\{C \in \mathcal{K}(X) \mid \int \frac{\log M_{e^{-s}}(C)}{s} d \Pi_{t}(s) \geq c\right\} .
\end{aligned}
$$

We must now prove that $F$ is closed. In order to show this, we fix a sequence $\left(C_{n}\right)_{n}$ in $F$ and $C \in \mathcal{K}(X)$ with $C_{n} \rightarrow C$. We must now prove that $C \in F$, i.e. we must show that

$$
\int \frac{\log M_{e^{-s}}(C)}{s} d \Pi_{t}(s) \geq c
$$

For brevity define functions $\varphi, \varphi_{n}:\left[t_{0}, \infty\right) \rightarrow[0, \infty)$ by $\varphi(s)=\frac{\log M_{e^{-s}}(C)}{s}$ and $\varphi_{n}(s)=$ $\frac{\log M_{e^{-s}}\left(C_{n}\right)}{s}$.

Claim 1. We have $\int \sup _{n} \varphi_{n} d \Pi_{t}<\infty$.

Proof of Claim 1. The measure $\Pi_{t}$ has compact support, and we can therefore choose $T_{0} \geq t_{0}$, such that $\operatorname{supp} \Pi_{t} \subseteq\left[t_{0}, T_{0}\right]$. Next, note that for all $n$ and

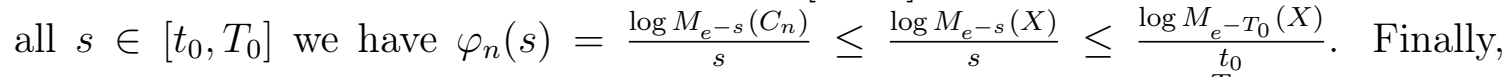
since $\operatorname{supp} \Pi_{t} \subseteq\left[t_{0}, T_{0}\right]$, we now conclude that $\int \sup _{n} \varphi_{n} d \Pi_{t}=\int_{t_{0}}^{T_{0}} \sup _{n} \varphi_{n} d \Pi_{t} \leq$ $\frac{\log M_{e}-T_{0}(X)}{t_{0}} \Pi_{t}\left(\left[t_{0}, T_{0}\right]\right)<\infty$ This completes the proof of Claim 1 . 
Claim 2. We have $c \leq \int \lim \sup _{n} \varphi_{n} d \Pi_{t}$.

Proof of Claim 2. Since $C_{n} \in F$, we conclude that $c \leq \int \frac{\log M_{e^{-s}}\left(C_{n}\right)}{s} d \Pi_{t}(s)=$ $\int \varphi_{n} d \Pi_{t}$ for all $n$, whence

$$
c \leq \limsup _{n} \int \varphi_{n} d \Pi_{t}
$$

We also note that it follows from Claim 1 and Lemma 6.1 (i.e. the reverse Fatou's Lemma) that

$$
\limsup _{n} \int \varphi_{n} d \Pi_{t} \leq \int \limsup _{n} \varphi_{n} d \Pi_{t} .
$$

The desired result now follows from (6.2) and (6.3). This completes the proof of Claim 2.

Claim 3. For all $s \geq t_{0}$, we have $\lim \sup _{n} \varphi_{n}(s) \leq \varphi(s)$.

Proof of Claim 3. This follows from the fact that $M_{r}: \mathcal{K}(X) \rightarrow \mathbf{R}$ is upper semi-continuous for all $r>0$ by Lemma 5.1. This completes the proof of Claim 3.

Finally, we deduce from Claim 2 and Claim 3 that

$$
c \leq \int \limsup _{n} \varphi_{n} d \Pi_{t} \leq \int \varphi d \Pi_{t}=\int \frac{\log M_{e^{-s}}(C)}{s} d \Pi_{t}(s) .
$$

This proves (6.1).

We now turn towards the proof of Theorem 2.2.(1).

Proof of Theorem 2.2.(1). Since clearly $\underline{\operatorname{dim}}_{\Pi, B}(C) \geq 0$, it suffices to prove that the set

$$
U=\left\{C \in \mathcal{K}(X) \mid \underline{\operatorname{dim}}_{\Pi, B}(C)>0\right\}
$$

is meagre. For $u>0$, write

$$
\left.U_{u}=\left\{C \in \mathcal{K}(X) \mid \underline{\operatorname{dim}}_{\Pi, B}(C)\right)>u\right\},
$$

and note that

$$
U=\bigcup_{u \in \mathbf{Q}, u>0} U_{u}
$$

It therefore suffices to show that $U_{u}$ is meagre for all $u \in \mathbf{Q}$ with $u>0$.

We therefore fix $u \in \mathbf{Q}$ with $u>0$. Next, in order to show that $U_{u}$ is meagre, we note that it suffices to show that there is a countable family $\left(G_{k}\right)_{k}$ of open and dense subsets of $\mathcal{K}(X)$ with $\cap_{k} G_{k} \subseteq \mathcal{K}(X) \backslash U_{u}$. We will now construct the sets $G_{k}$. For $t \geq t_{0}$, let

$$
L_{t}=\left\{C \in \mathcal{K}(X) \mid \int \frac{\log M_{e^{-s}}(C)}{s} d \Pi_{t}(s)<u\right\}
$$

and for a positive integer $k$, put

$$
G_{k}=\bigcup_{t \geq k} L_{t} .
$$

Below we show that the family $\left(G_{k}\right)_{k}$ consists of open and dense subsets of $\mathcal{K}(X)$ with $\bigcap_{k} G_{k} \subseteq \mathcal{K}(X) \backslash U_{u}$; this is the contents of the following three claims.

Claim 1. The set $G_{n}$ is open in $\mathcal{K}(X)$. 
Proof of Claim 1. Indeed, since it follows from Lemma 6.2 that $L_{t}$ is open for all $t \geq t_{0}$, we immediately conclude that $G_{k}=\bigcup_{t \geq k} L_{t}$ is open. This completes the proof of Claim 1.

Claim 2. The set $G_{k}$ is dense in $\mathcal{K}(X)$.

Proof of Claim 2. Let $C \in \mathcal{K}(X)$ and let $r>0$. We must now find $K \in \mathcal{K}(X)$ such that $d_{H}(C, K)<r$ and $K \in G_{n}$. It is clear that we can choose a finite, and hence compact, subset $K$ of $X$ such that $d_{H}(C, K)<r$. We now show that $K \in G_{k}$. Indeed, since $K$ is finite, we conclude that $\frac{\log M_{e^{-t}}(K)}{t} \rightarrow 0$ as $t \rightarrow \infty$, and the consistency condition therefore implies that $\int \frac{\log M_{e-s}(K)}{s} d \Pi_{t}(s) \rightarrow 0$ as $t \rightarrow \infty$. We conclude immediately from that there is a real number $t \geq n$ such that $\int \frac{\log M_{e^{-s}}(K)}{s} d \Pi_{t}(s) \leq u$, and so $K \in L_{t} \subseteq G_{n}$. This completes the proof of Claim 2 .

Claim 3. We have $\bigcap_{n} G_{n} \subseteq \mathcal{K}(X) \backslash U_{u}$.

Proof of Claim 3. Let $C \in \bigcap_{n} G_{n}$. Hence for each positive integer $n$, we can find $t_{n} \geq n$ such that $C \in L_{t_{n}}$, whence $\int \frac{\log M_{e^{-s}}(C)}{s} d \Pi_{t_{n}}(s)<u$ for all positive integers $n$, and so $\liminf \operatorname{int}_{t \rightarrow \infty} \int \frac{\log M_{e^{-s}}(C)}{s} d \Pi_{t}(s) \leq \liminf _{n} \int \frac{\log M_{e^{-s}}(C)}{s} d \Pi_{t_{n}}(s) \leq u$. It follows immediately from this and Lemma 5.2 that $\underline{\operatorname{dim}}_{\Pi, B}(C)=\liminf _{t \rightarrow \infty} \int \frac{\log N_{e^{-s}}(C)}{s} d \Pi_{t}(s)$ $\leq \liminf _{t \rightarrow \infty} \int \frac{\log M_{e^{-s}}(C)}{s} d \Pi_{t}(s) \leq u$, whence $C \in \mathcal{K}(X) \backslash U_{u}$. This completes the proof of Claim 3.

Combining Claims 1, 2 and 3, we now conclude that $U_{u}$ is meagre.

\section{Proof of the upper bound: Theorem 2.2.(2)}

The purpose of this section is to prove Theorem 2.2.(2). Recall that the notation $\mathcal{K}(X ; x, r), \mathcal{K}_{\Pi, s}(X ; x, r), \Delta_{\Pi}(x, r)$ and $\Delta_{\Pi}(x)$ is defined in the statement of Theorem 2.2. In addition, we will use the following notation throughout this section. Namely, if $(X, d)$ is a metric space and $E, F \subseteq X$, the we write

$$
\operatorname{dist}(E, F)=\inf _{x \in E, y \in F} d(x, y) .
$$

We first prove three auxiliary lemmas.

Lemma 7.1. Let $X$ be a metric space and let $\Pi=\left(\Pi_{t}\right)_{t \geq t_{0}}$ be an averaging system. Let $x, y \in X$ and $\rho, \sigma>0$ and assume that $B(x, \rho) \subseteq B(y, \sigma)$.

(1) Let $s \geq 0$. If $\overline{\mathcal{K}_{\Pi, s}(X ; y, \sigma)}=\mathcal{K}(X ; y, \sigma)$, then $\overline{\mathcal{K}_{\Pi, s}(X ; x, \rho)}=\mathcal{K}(X ; x, \rho)$.

(2) We have $\Delta_{\Pi}(x, \rho) \geq \Delta_{\Pi}(y, \sigma)$.

Proof. (1) Let $C \in \mathcal{K}(X ; x, \rho)$ and $r>0$. We must now find $K \in \mathcal{K}_{\Pi, s}(X ; x, \rho)$ such that $d_{H}(C, K)<r$. We first prove the following claim.

Claim 1. There is $L \in \mathcal{K}(X)$ such that $L \subseteq B(x, \rho)$ and $d_{H}(C, L)<\frac{r}{2}$.

Proof of Claim 1. Let $d$ denote the metric in $X$. Since $C \in \mathcal{K}(X ; x, \rho)$, we conclude that $C \subseteq \overline{B(x, \rho)}$. It follows from this that for each $x \in C$, we can choose $y_{x} \in B(x, r)$ such that $d\left(x, y_{x}\right)<\frac{r}{2}$. It is clear that $C \subseteq \bigcup_{x \in C} B\left(y_{x}, \frac{r}{2}\right)$, and it therefore follows from the compactness of $C$ that there is a finite (and hence compact) subset $E$ of $C$ such that $C \subseteq \bigcup_{x \in E} B\left(y_{x}, \frac{r}{2}\right)$. Now put $L=\left\{y_{x} \mid x \in E\right\}$, and note that it follows from the construction of $L$ that $L \subseteq B(x, \rho)$ and $d_{H}(C, L)<\frac{r}{2}$. This completes the proof of Claim 1. 
Let $L \in \mathcal{K}(X)$ be the set from Claim 1. Next, note that

$$
l=\operatorname{dist}(L, X \backslash B(x, \rho))>0 .
$$

Since also $L \subseteq B(x, \rho) \subseteq B(y, \sigma) \subseteq \overline{B(y, \sigma)}$, we conclude that $L \in \mathcal{K}(X ; y, \sigma)=$ $\overline{\mathcal{K}_{\Pi, s}(X ; y, \sigma)}$, and we can therefore choose $K \in \mathcal{K}_{\Pi, s}(X ; y, \sigma)$ with

$$
d_{H}(L, K)<\min \left(\frac{r}{2}, \frac{l}{2}\right) .
$$

We now claim that

$$
\begin{aligned}
& K \in \mathcal{K}_{\Pi, s}(X ; x, \rho), \\
& d_{H}(C, K)<r .
\end{aligned}
$$

Proof of (7.1). $K \in \mathcal{K}_{\Pi, s}(X ; x, \rho)$. We first show that $K \subseteq \overline{B(x, r)}$. Indeed, since $d_{H}(L, K)<\min \left(\frac{r}{2}, \frac{l}{2}\right) \leq \frac{l}{2}=\frac{1}{2} \operatorname{dist}(L, X \backslash B(x, \rho))$, we deduce that $K \subseteq B(x, \rho) \subseteq$ $\overline{B(x, \rho)}$. Next, we show that $\overline{\operatorname{dim}}_{\Pi, B}(K) \geq s$. However, this follows from the fact that $K \in \mathcal{K}_{\Pi, s}(X ; y, \sigma)$. This completes the proof of (7.1).

Proof of (7.2). $d_{H}(C, K)<r$. This follows from the fact that $d_{H}(C, K) \leq$ $d_{H}(C, L)+d_{H}(L, K)<\frac{r}{2}+\frac{r}{2}=r$. This completes the proof of $(7.2)$.

Finally, the desired statement follows from (7.1)-(7.2).

(2) This statement follows immediately from (1).

Lemma 7.2. Let $X$ be a metric space and let $\Pi=\left(\Pi_{t}\right)_{t \geq t_{0}}$ be an averaging system. Let $c \in \mathbf{R}$ and $t \geq t_{0}$. Then the set

$$
\left\{C \in \mathcal{K}(X) \mid \int \frac{\log N_{e^{-s}}(C)}{s} d \Pi_{t}(s)>c\right\}
$$

is open in $\mathcal{K}(X)$.

Proof. Write

$$
\begin{aligned}
F & =\mathcal{K}(X) \backslash\left\{C \in \mathcal{K}(X) \mid \int \frac{\log N_{e^{-s}}(C)}{s} d \Pi_{t}(s)>c\right\} \\
& =\left\{f \in \mathcal{K}(X) \mid \int \frac{\log N_{e^{-s}}(C)}{s} d \Pi_{t}(s) \leq c\right\} .
\end{aligned}
$$

We must now prove that $F$ is closed. In order to show this, we fix a sequence $\left(C_{n}\right)_{n}$ in $F$ and $C \in \mathcal{K}(X)$ with $C_{n} \rightarrow C$. We must now prove that $f \in F$, i.e. we must show that

$$
\int \frac{\log N_{e^{-s}}(C)}{s} d \Pi_{t}(s) \leq c
$$

For brevity define functions $\varphi, \varphi_{n}:\left[t_{0}, \infty\right) \rightarrow[0, \infty)$ by $\varphi(s)=\frac{\log N_{e^{-s}}(C)}{s}$ and $\varphi_{n}(s)=$ $\frac{\log N_{e^{-s}}(C)}{s}$.

Claim 1. We have $\int \liminf _{n} \varphi_{n} d \Pi_{t} \leq c$.

Proof of Claim 1. Since $C_{n} \in F$, we conclude that $\int \varphi_{n} d \Pi_{t}=\int \frac{\log N_{e^{-s}}(C)}{s} d \Pi_{t}(s) \leq$ $c$ for all $n$, whence

$$
\liminf _{n} \int \varphi_{n} d \Pi_{t} \leq c
$$


We also note that it follows from Fatou's lemma that

$$
\int \liminf _{n} \varphi_{n} d \Pi_{t} \leq \liminf _{n} \int \varphi_{n} d \Pi_{t} .
$$

The desired result now follows from (7.4) and (7.5). This competes the proof of Claim 2.

Claim 2. For all $s \geq t_{0}$, we have $\varphi(s) \leq \liminf _{n} \varphi_{n}(s)$.

Proof of Claim 2. This follows from the fact that map $N_{r}: \mathcal{K}(X) \rightarrow \mathbf{R}$ is lower semi-continuous for all $r>0$ by Lemma 5.1. This completes the proof of Claim 2.

Finally, we deduce from Claim 1 and Claim 2 that

$$
\int \frac{\log N_{e^{-s}}(C)}{s} d \Pi_{t}(s)=\int \varphi d \Pi_{t} \leq \int \liminf _{n} \varphi_{n} d \Pi_{t} \leq c .
$$

This proves (7.3).

Lemma 7.3. Let $X$ be a metric space. If $C_{1}, \ldots, C_{n}, K_{1}, \ldots, K_{n} \in \mathcal{K}(X)$ and $L \in \mathcal{K}(X) \cup\{\varnothing\}$, then

$$
d_{H}\left(L \cup \bigcup_{i} C_{i}, L \cup \bigcup_{i} K_{i}\right) \leq \max _{i} d_{H}\left(C_{i}, K_{i}\right) .
$$

Proof. This follows easily from the definition of the Hausdorff metric.

We now turn towards the proof of Theorem 2.2.(2). The proof of Theorem 2.2.(2) is based on Proposition 7.4 and Proposition 7.5 presented below.

Proposition 7.4. Let $X$ be a metric space and let $\Pi=\left(\Pi_{t}\right)_{t \geq t_{0}}$ be an averaging system. Let $\left(x_{n}\right)_{n}$ be a sequence of points from $X$ and let $\left(r_{n}\right)_{n}$ be sequence of positive real numbers.

(1) For $n \in \mathbf{N}$, let

$$
\begin{aligned}
T_{n}=\{C \in \mathcal{K}(X) \mid & \text { for all } i=1, \ldots, n, \text { we have } \\
& \left.\overline{\operatorname{dim}}_{\Pi, B}\left(C \cap \overline{B\left(x_{i}, 5 r_{n}\right)}\right) \geq \Delta_{\Pi}\left(x_{i}, r_{n}\right)\right\} .
\end{aligned}
$$

Then the set $T_{n}$ is co-meagre in $\mathcal{K}(X)$.

(2) Let

$$
\begin{gathered}
T=\{C \in \mathcal{K}(X) \mid \text { for all } n \in \mathbf{N} \text { and for all } i=1, \ldots, n, \text { we have } \\
\left.\qquad \overline{\operatorname{dim}}_{\Pi, B}\left(C \cap \overline{B\left(x_{i}, 5 r_{n}\right)}\right) \geq \Delta_{\Pi}\left(x_{i}, r_{n}\right)\right\} .
\end{gathered}
$$

Then the set $T$ is co-meagre in $\mathcal{K}(X)$.

Proof. (1) Let $d$ denote the metric in $X$. We must prove that the set

$$
\begin{aligned}
& U=\mathcal{K}(X) \backslash T_{n}=\{C \in \mathcal{K}(X) \mid \text { there is an } i=1, \ldots, n \text { such that } \\
&\left.\overline{\operatorname{dim}}_{\Pi, B}\left(C \cap \overline{B\left(x_{i}, 5 r_{n}\right)}\right)<\Delta_{\Pi}\left(x_{i}, r_{n}\right)\right\}
\end{aligned}
$$

is meagre.

For $u>0$, write

$$
\begin{aligned}
U_{u}=\{C \in \mathcal{K}(X) \mid & \text { there is an } i=1, \ldots, n \text { such that } \\
& \left.\overline{\operatorname{dim}}_{\Pi, B}\left(C \cap \overline{B\left(x_{i}, 5 r_{n}\right)}\right)<\Delta_{\Pi}\left(x_{i}, r_{n}\right)-u\right\} .
\end{aligned}
$$

Since

$$
U=\bigcup_{u \in \mathbf{Q}, u>0} U_{u}
$$


it clearly suffices to show that $U_{u}$ is meagre for all $u \in \mathbf{Q}$ with $u>0$. We therefore fix $u \in \mathbf{Q}$ with $u>0$, and note that it suffices to show that there is a countable family $\left(G_{k}\right)_{k}$ of open and dense subsets of $\mathcal{K}(X)$ with $\cap_{k} G_{k} \subseteq \mathcal{K}(X) \backslash U_{u}$.

For $i=1, \ldots, n$ and $t \geq t_{0}$, let

$$
\Lambda_{t, i}=\left\{C \in \mathcal{K}(X) \mid \int \frac{\log N_{e^{-s}}(C)}{s} d \Pi_{t}(s)>\Delta_{\Pi}\left(x_{i}, r_{n}\right)-u\right\},
$$

and for $t_{1}, \ldots, t_{n} \geq t_{0}$, put

$$
\begin{aligned}
L_{t_{1}, \ldots, t_{n}}= & \left\{C_{0} \cup C_{1} \cup \ldots \cup C_{n} \mid C_{0} \in \mathcal{K}(X) \cup\{\varnothing\}, C_{0} \subseteq X \backslash \bigcup_{i=1}^{n} \overline{B\left(x_{i}, r_{n}\right)},\right. \\
& \text { for all } i=1, \ldots, n, \text { we have } C_{i} \in \Lambda_{t_{i}, i}, \\
& \text { for all } \left.i=1, \ldots, n, \text { we have } C_{i} \subseteq B\left(x_{i}, 5 r_{n}\right)\right\} .
\end{aligned}
$$

Finally, for a positive integer $k$, put

$$
G_{k}=\bigcup_{t_{1}, \ldots, t_{n} \geq k} L_{t_{1}, \ldots, t_{n}}
$$

Below we show that the family $\left(G_{k}\right)_{k}$ consists of open and dense subsets of $\mathcal{K}(X)$ with $\bigcap_{k} G_{k} \subseteq C_{u}(X) \backslash U_{u}$; this is the contents of the following four claims.

Claim 1. The set $L_{t_{1}, \ldots, t_{n}}$ is open in $\mathcal{K}(X)$.

Proof of Claim 1. Let $C \in L_{t_{1}, \ldots, t_{n}}$. We must now find $r>0$ such that $B(C, r) \subseteq$ $L_{t_{1}, \ldots, t_{n}}$. We first note that since $C \in L_{t_{1}, \ldots, t_{n}}$, there are sets $C_{0}, C_{1}, \ldots, C_{n}$ with

$$
C=C_{0} \cup C_{1} \cup \ldots \cup C_{n}
$$

such that

$$
\begin{aligned}
& C_{0} \in \mathcal{K}(X) \cup\{\varnothing\}, \\
& C_{0} \subseteq X \backslash \bigcup_{i=1}^{n} \overline{B\left(x_{i}, r_{n}\right)}, \\
& \text { for all } i=1, \ldots, n, \text { we have } C_{i} \in \Lambda_{t_{i}, i}, \\
& \text { for all } i=1, \ldots, n, \text { we have } C_{i} \subseteq B\left(x_{i}, 5 r_{n}\right) .
\end{aligned}
$$

Let

$$
d_{0}= \begin{cases}\operatorname{dist}\left(C_{0}, \cup_{i=1}^{n} \overline{B\left(x_{i}, r_{n}\right)}\right) & \text { if } C_{0} \neq \varnothing \\ \infty & \text { if } C_{0}=\varnothing\end{cases}
$$

and note that if $C_{0} \neq \varnothing$, then $C_{0} \subseteq X \backslash \bigcup_{i=1}^{n} \overline{B\left(x_{i}, r_{n}\right)}$ and $C_{0}$ is compact, whence $d_{0}=\operatorname{dist}\left(C_{0}, \bigcup_{i=1}^{n} \overline{B\left(x_{i}, r_{n}\right)}\right)>0$. Also, since $C_{i} \in \Lambda_{t_{i}, i}$ for all $i=1, \ldots, n$ and $\Lambda_{t_{i}, i}$ is open (by Lemma 7.2), we conclude that there is a positive number $\rho_{i}>0$ such that $B\left(C_{i}, \rho_{i}\right) \subseteq \Lambda_{t_{i}, i}$. Finally, since $C_{i} \subseteq B\left(x_{i}, 5 r_{n}\right)$ for all $i=1, \ldots, n$ and $C_{i}$ is compact, we conclude that $d_{i}=\operatorname{dist}\left(C_{i}, X \backslash B\left(x_{i}, 5 r_{n}\right)\right)>0$. Now put

$$
r=\min _{i=1, \ldots, n}\left(\frac{d_{0}}{4}, \frac{\rho_{i}}{4}, \frac{d_{i}}{4}, \frac{r_{n}}{8}\right) \text {. }
$$

We claim that

$$
B(C, r) \subseteq L_{t_{1}, \ldots, t_{n}} .
$$

We will now prove (7.6). We therefore let $K \in B(C, r)$. We must now prove that $K \in L_{t_{1}, \ldots, t_{n}}$, i.e. we must show that there are sets $K_{0}, K_{1}, \ldots, K_{n}$ with

$$
K=K_{0} \cup K_{1} \cup \ldots \cup K_{n}
$$


such that

$$
\begin{aligned}
& K_{0} \in \mathcal{K}(X) \cup\{\varnothing\}, \\
& K_{0} \subseteq X \backslash \cup_{i=1}^{n} \overline{B\left(x_{i}, r_{n}\right)}, \\
& \text { for all } i=1, \ldots, n, \text { we have } K_{i} \in \Lambda_{t_{i}, i}, \\
& \text { for all } i=1, \ldots, n, \text { we have } K_{i} \subseteq B\left(x_{i}, 5 r_{n}\right) .
\end{aligned}
$$

Since $C_{i}$ is compact and $C_{i} \subseteq \bigcup_{x \in C_{i}} B(x, r)$, we can find a finite subset $E_{i}$ of $C_{i}$ such that

$$
C_{i}=\bigcup_{x \in E_{i}} B(x, r) \subseteq \bigcup_{x \in E_{i}} \overline{B(x, r)}
$$

for $i=0,1, \ldots, n$. Now put

$$
K_{i}=K \cap \bigcup_{x \in E_{i}} \overline{B(x, 2 r)}
$$

We now show that the sets $K_{0}, K_{1}, \ldots, K_{n}$ satisfy (7.7)-(7.11).

Proof of (7.7). $K=K_{0} \cup K_{1} \cup \ldots \cup K_{n}$. It is clear that $K_{0} \cup K_{1} \cup \ldots \cup K_{n} \subseteq K$ and it therefore suffices to prove that $K \subseteq K_{0} \cup K_{1} \cup \ldots \cup K_{n}$. We therefore fix $x \in K$. Since $d_{H}(C, K)<r$, there is a point $y \in C$ such that $d(x, y)<r$. Also, since $y \in C$, there is an index $i=0,1, \ldots, n$ such that $y \in C_{i} \subseteq \bigcup_{z \in E_{i}} B(z, r)$. We conclude from this that $x \in \bigcup_{z \in E_{i}} B(z, 2 r) \subseteq \bigcup_{z \in E_{i}} \overline{B(z, 2 r)}$, and so $x \in K \cap \bigcup_{z \in E_{i}} \overline{B(z, 2 r)}=K_{i}$. This completes the proof of (7.7).

Proof of (7.8). $K_{0} \in \mathcal{K}(X) \cup\{\varnothing\}$. If $K_{0}=\varnothing$, then the assertion is clear, so we may assume that $K_{0} \neq \varnothing$. In this case $K_{0}$ is compact since $K$ is compact and $\bigcup_{z \in E_{0}} \overline{B(z, 2 r)}$ is closed (because $E_{0}$ is finite), whence $K_{0} \in \mathcal{K}(X)$. This completes the proof of (7.8).

Proof of (7.9). $K_{0} \subseteq X \backslash \bigcup_{i=1}^{n} \overline{B\left(x_{i}, r_{n}\right)}$. If $K_{0}=\varnothing$, then the assertion is clear, so we may assume that $K_{0} \neq \varnothing$. In this case $K_{0}=K \cap \bigcup_{x \in E_{0}} \overline{B(x, 2 r)} \subseteq \bigcup_{x \in C_{0}} \overline{B(x, 2 r)}$ where $2 r<\frac{d_{0}}{2}=\frac{1}{2} \operatorname{dist}\left(C_{0}, \bigcup_{i=1}^{n} \overline{B\left(x_{i}, r_{n}\right)}\right)$, and so $K_{0} \subseteq X \backslash \bigcup_{i=1}^{n} \overline{B\left(x_{i}, r_{n}\right)}$. This completes the proof of (7.9).

Proof of (7.10). For all $i=1, \ldots, n$, we have $K_{i} \in \Lambda_{t_{i}, i}$. We first note that $K_{i}$ is compact. Indeed, this is clear since $K$ is compact and $\bigcup_{z \in E_{i}} \overline{B(z, 2 r)}$ is closed (because $E_{i}$ is finite). Next, we prove that $K_{i} \neq \varnothing$. In order to prove this we first choose a point $x \in E_{i}$, and note that since $x \in E_{i} \subseteq C_{i} \subseteq C$ and $d_{H}(C, K)<r$, we can find a point $y \in K$ such that $d(x, y)<r$. We conclude from this that $y \in K \cap \bigcup_{z \in E_{i}} B(z, r) \subseteq K \cap \bigcup_{z \in E_{i}} \overline{B(z, 2 r)}=K_{i}$, and so $K_{i} \neq \varnothing$.

Next, we show that $K_{i} \in L_{t_{i}, i}$. We first show that

$$
C \cap \overline{B(x, r)} \neq \varnothing \quad \text { and } \quad K \cap \overline{B(x, 2 r)} \neq \varnothing
$$

for all $i=1, \ldots, n$ and for all $x \in E_{i}$. Indeed, it is clear that $C \cap \overline{B(x, r)} \neq \varnothing$ because $x \in E_{i} \subseteq C_{i} \subseteq C$ and $x \in \overline{B(x, r)}$, and since $d_{H}(C, K)<r$ and $x \in E_{i} \subseteq C_{i} \subseteq C$, we deduce that there is a point $y \in K$ such that $d(x, y)<r$, whence $y \in K \cap \overline{B(x, 2 r)}$. This completes the proof of (7.12). 
It follows from (7.12) that $C \cap \overline{B(x, r)}, K \cap \overline{B(x, 2 r)} \in \mathcal{K}(X)$ for all $i=1, \ldots, n$ and for all $x \in E_{i}$, and Lemma 7.3 therefore implies that

$$
\begin{aligned}
d_{H}\left(C_{i}, K_{i}\right) & =d_{H}\left(\bigcup_{x \in E_{i}} C \cap \overline{B(x, r)}, \bigcup_{x \in E_{i}} K \cap \overline{B(x, 2 r)}\right) \\
& \leq \max _{x \in E_{i}} d_{H}(C \cap \overline{B(x, r)}, K \cap \overline{B(x, 2 r)}) \leq \max _{x \in E_{i}} 2 r=2 r<\rho_{i} .
\end{aligned}
$$

Finally, we deduce from (7.13) that $K_{i} \in B\left(C_{i}, \rho_{i}\right) \subseteq \Lambda_{t_{i}, i}$. This completes the proof of (7.10).

Proof of (7.11). For all $i=1, \ldots, n$, we have $K_{i} \subseteq \overline{B\left(x_{i}, 5 r_{n}\right)}$. We have $K_{i}=$ $K \cap \cup_{x \in E_{i}} \overline{B(x, 2 r)} \subseteq \cup_{x \in C_{i}} \overline{B(x, 2 r)}$ where $2 r<\frac{d_{i}}{2}=\frac{1}{2} \operatorname{dist}\left(C_{i}, X \backslash B\left(x_{i}, 5 r_{n}\right)\right)$. This clearly implies that $K_{i} \subseteq B\left(x_{i}, 2 r_{n}\right) \subseteq \overline{B\left(x_{i}, 2 r_{n}\right)}$. This completes the proof of (7.11).

The statement in Claim 1 follows from (7.7)-(7.11). This completes the proof of Claim 1.

Claim 2. The set $G_{k}$ is open in $\mathcal{K}(X)$.

Proof of Claim 2. This follows immediately from Claim 1. This completes the proof of Claim 2.

Claim 3. The set $G_{k}$ is dense in $\mathcal{K}(X)$.

Proof of Claim 3. Let $C \in \mathcal{K}(X)$ and let $r>0$. We must now find $K \in G_{k}$ such that $d_{H}(C, K)<r$, i.e. we must show that there are sets $K_{0}, K_{1}, \ldots, K_{n}$ and numbers $t_{1}, \ldots, t_{n}$ with $t_{1}, \ldots, t_{n} \geq k$ such that if we put

$$
K=K_{0} \cup K_{1} \cup \ldots \cup K_{n},
$$

then

$$
\begin{aligned}
& K_{0} \in \mathcal{K}(X) \cup\{\varnothing\}, \\
& K_{0} \subseteq X \backslash \bigcup_{i=1}^{n} \overline{B\left(x_{i}, r_{n}\right)}, \\
& \text { for all } i=1, \ldots, n, \text { we have } K_{i} \in \Lambda_{t_{i}, i}, \\
& \text { for all } i=1, \ldots, n, \text { we have } K_{i} \subseteq B\left(x_{i}, 5 r_{n}\right),
\end{aligned}
$$

and

$$
d_{H}(C, K)<r
$$

Fix $i=1, \ldots, n$. Since $\Delta_{\Pi}\left(x_{i}, r_{n}\right)>\Delta_{\Pi}\left(x_{i}, r_{n}\right)-\frac{u}{2}$, we conclude that

$$
\overline{\mathcal{K}_{\Pi, \Delta_{\Pi}\left(x_{i}, r_{n}\right)-\frac{u}{2}}\left(X ; x_{i}, r_{n}\right)}=\mathcal{K}_{\Pi}\left(X ; x_{i}, r_{n}\right),
$$

and we can therefore find a compact set $C_{i} \in \mathcal{K}_{\Pi, \Delta_{\Pi}\left(x_{i}, r_{n}\right)-\frac{u}{2}}\left(X ; x_{i}, r_{n}\right)$ with

$$
d_{H}\left(C_{i}, C \cap \overline{B\left(x_{i}, r_{n}\right)}\right)<r .
$$

Also, since $C_{i} \in \mathcal{K}_{\Pi, \Delta_{\Pi}\left(x_{i}, r_{n}\right)-\frac{u}{2}}\left(X ; x_{i}, r_{n}\right)$, we have

$$
\begin{aligned}
C_{i} & \subseteq \overline{B\left(x_{i}, r_{n}\right)}, \\
\overline{\operatorname{dim}}_{\Pi, B}\left(C_{i}\right) & \geq \Delta_{\Pi}\left(x_{i}, r_{n}\right)-\frac{u}{2} .
\end{aligned}
$$

It follows from (7.21) that

$$
\limsup _{t} \int \frac{\log N_{e^{-s}}\left(C_{i}\right)}{s} d \Pi_{t}(s) \geq \overline{\operatorname{dim}}_{\Pi, B}\left(C_{i}\right) \geq \Delta_{\Pi}\left(x_{i}, r_{n}\right)-\frac{u}{2}>\Delta_{\Pi}\left(x_{i}, r_{n}\right)-u
$$


and we can therefore find $t_{i} \geq k$ with

$$
\int \frac{\log N_{e^{-s}}\left(C_{i}\right)}{s} d \Pi_{t_{i}}(s)>\Delta_{\Pi}\left(x_{i}, r_{n}\right)-u .
$$

Next, put

$$
K_{0}=C \cap\left(X \backslash \bigcup_{i=1}^{n} B\left(x_{i}, 2 r_{n}\right)\right),
$$

and for $i=1, \ldots, n$, put

$$
S_{i}=C \cap\left(\overline{B\left(x_{i}, 4 r_{n}\right)} \backslash B\left(x_{i}, r_{n}\right)\right)
$$

and

$$
K_{i}=C_{i} \cup S_{i}
$$

Finally, let

$$
K=K_{0} \cup K_{1} \cup \ldots \cup K_{n} .
$$

We now show that the sets $K_{0}, K_{1}, \ldots, K_{n}$ and the numbers $t_{1}, \ldots, t_{n}$ satisfy (7.14)(7.18).

Proof of (7.14). $K_{0} \in \mathcal{K}(X) \cup\{\varnothing\}$. We divide the proof into two cases depending on whether $K_{0}$ is empty or not. If $K_{0}=\varnothing$, then the assertion is clear. Assume now that $K_{0} \neq \varnothing$. In this case $K_{0}$ is compact since $K$ is compact and $X \backslash \bigcup_{i=1}^{n} B\left(x_{i}, 2 r_{n}\right)$ is closed, whence $K_{0} \in \mathcal{K}(X)$. This completes the proof of (7.14).

Proof of (7.15). $K_{0} \subseteq X \backslash \bigcup_{i=1}^{n} \overline{B\left(x_{i}, r_{n}\right)}$. We have $K_{0}=C \cap\left(X \backslash \bigcup_{i=1}^{n} B\left(x_{i}, 2 r_{n}\right)\right)$ $\subseteq X \backslash \bigcup_{i=1}^{n} \overline{B\left(x_{i}, r_{n}\right)}$. This completes the proof of (7.15).

Proof of (7.16). For all $i=1, \ldots, n$, we have $K_{i} \in \Lambda_{t_{i}, i}$. We first note that $K_{i}$ is compact. Indeed, since $C$ is compact and $\overline{B\left(x_{i}, 4 r_{n}\right)} \backslash B\left(x_{i}, r_{n}\right)$ is closed, we conclude that $S_{i}$ is compact. This clearly implies that $K_{i}=C_{i} \cup S_{i}$ is compact. Next, we observe that $K_{i} \neq \varnothing$. However, this follows immediately from the fact that $K_{i} \supseteq C_{i}$ and $C_{i} \neq \varnothing$ (because $\left.C_{i} \in \mathcal{K}_{\Pi, \Delta_{\Pi}\left(x_{i}, r_{n}\right)-\frac{u}{2}}\left(X ; x_{i}, r_{n}\right) \subseteq \mathcal{K}(X)\right)$. We therefore conclude that $K_{i} \in \mathcal{K}(X)$.

Finally, we show that $K_{i} \in \Lambda_{t_{i}, i}$. Since $K_{i} \supseteq C_{i}$, we conclude from (7.22) that

$$
\int \frac{\log N_{e^{-s}}\left(K_{i}\right)}{s} d \Pi_{t_{i}}(s) \geq \int \frac{\log N_{e^{-s}}\left(C_{i}\right)}{s} d \Pi_{t_{i}}(s)>\Delta_{\Pi}\left(x_{i}, r_{n}\right)-u .
$$

This completes the proof of (7.16).

Proof of (7.17). For all $i=1, \ldots, n$, we have $K_{i} \subseteq \overline{B\left(x_{i}, 5 r_{n}\right)}$. We have $K_{i}=$

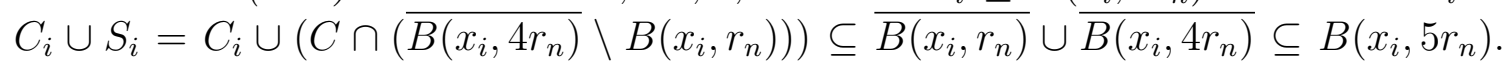
This completes the proof of (7.17).

Proof of (7.18). $d_{H}(C, K)<r$. Since $X=\left(X \backslash \bigcup_{i=1}^{n} B\left(x_{i}, 2 r_{n}\right)\right) \cup \bigcup_{i=1}^{n} \overline{B\left(x_{i}, 4 r_{n}\right)}$, we conclude that $C=\left(C \cap\left(X \backslash \bigcup_{i=1}^{n} B\left(x_{i}, 2 r_{n}\right)\right)\right) \cup \bigcup_{i=1}^{n}\left(C \cap \overline{B\left(x_{i} 4 r_{n}\right)}\right)$, whence

$$
d_{H}(C, K)=d_{H}\left(\left(C \cap\left(X \backslash \bigcup_{i=1}^{n} B\left(x_{i}, 2 r_{n}\right)\right)\right) \cup \bigcup_{i=1}^{n}\left(C \cap \overline{B\left(x_{i} 4 r_{n}\right)}\right), K_{0} \cup \bigcup_{i=1}^{n} K_{i}\right) .
$$

However, since $K_{0}=C \cap\left(X \backslash \bigcup_{i=1}^{n} B\left(x_{i}, 2 r_{n}\right)\right)$, the above expression for $d_{H}(C, K)$ simplifies to

$$
d_{H}(C, K)=d_{H}\left(K_{0} \cup \bigcup_{i=1}^{n}\left(C \cap \overline{B\left(x_{i}, 4 r_{n}\right)}\right), K_{0} \cup \bigcup_{i=1}^{n} K_{i}\right) .
$$


Since $C \cap \overline{B\left(x_{i}, 4 r_{n}\right)}, K_{i} \in \mathcal{K}(X)$ for $i=1, \ldots, n$ and $K_{0} \in \mathcal{K}(X) \cup\{\varnothing\}$, we conclude from (7.23) and Lemma 7.3 that

$$
\begin{aligned}
d_{H}(C, K) & \leq d_{H}\left(K_{0} \cup \bigcup_{i=1}^{n}\left(C \cap \overline{B\left(x_{i}, 4 r_{n}\right)}\right), K_{0} \cup \bigcup_{i=1}^{n} K_{i}\right) \\
& \leq \max _{i=1, \ldots, n} d_{H}\left(C \cap \overline{B\left(x_{i}, 4 r_{n}\right)}, K_{i}\right) .
\end{aligned}
$$

Since clearly $C \cap \overline{B\left(x_{i}, 4 r_{n}\right)}=\left(C \cap \overline{B\left(x_{i}, r_{n}\right)}\right) \cup\left(C \cap\left(\overline{B\left(x_{i}, 4 r_{n}\right)} \backslash B\left(x_{i}, r_{n}\right)\right)\right)$, we conclude from (7.24) that

$$
\begin{aligned}
& d_{H}(C, K) \leq \max _{i=1, \ldots, n} d_{H}\left(C \cap \overline{B\left(x_{i}, 4 r_{n}\right)}, K_{i}\right) \\
& =\max _{i=1, \ldots, n} d_{H}\left(\left(C \cap \overline{B\left(x_{i}, r_{n}\right)}\right) \cup\left(C \cap\left(\overline{B\left(x_{i}, 4 r_{n}\right)} \backslash B\left(x_{i}, r_{n}\right)\right)\right), C_{i} \cup S_{i}\right) .
\end{aligned}
$$

Using the fact that $S_{i}=C \cap\left(\overline{B\left(x_{i}, 4 r_{n}\right)} \backslash B\left(x_{i}, r_{n}\right)\right)$, it follows from (7.25) that

$$
d_{H}(C, K) \leq \max _{i=1, \ldots, n} d_{H}\left(\left(C \cap \overline{B\left(x_{i}, r_{n}\right)}\right) \cup S_{i}, C_{i} \cup S_{i}\right)
$$

Next, since $C \cap \overline{B\left(x_{i}, r_{n}\right)}, C_{i} \in \mathcal{K}(X)$ for $i=1, \ldots, n$ and $S_{i} \in \mathcal{K}(X) \cup\{\varnothing\}$, we now deduce from the above and Lemma 7.3 that

$$
\begin{aligned}
d_{H}(C, K) & \leq \max _{i=1, \ldots, n} d_{H}\left(\left(C \cap \overline{B\left(x_{i}, r_{n}\right)}\right) \cup S_{i}, C_{i} \cup S_{i}\right) \\
& \leq \max _{i=1, \ldots, n} d_{H}\left(C \cap \overline{B\left(x_{i}, r_{n}\right)}, C_{i}\right) .
\end{aligned}
$$

Finally, since $d_{H}\left(C \cap \overline{B\left(x_{i} r_{n}\right)}, C_{i}\right)<r$ for all $i=1, \ldots, n$ (by (7.19)), it follows from (7.26) that $d_{H}(C, K)<r$. This completes the proof of (7.18).

The statement in Claim 3 follows from (7.14)-(7.18). This completes the proof of Claim 3.

Claim 4. We have $\bigcap_{k} G_{k} \subseteq \mathcal{K}(X) \backslash U_{u}$.

Proof of Claim 4. Let $C \in \bigcap_{k} G_{k}$. Hence for each positive integer $k$, we can find real numbers $t_{k, 1}, \ldots, t_{k, n}$ with $t_{k, 1}, \ldots, t_{k, n} \geq k$ such that $C \in L_{t_{k, 1}, \ldots, t_{k, n}}$. In particular, this implies that there are sets $C_{k, 0}, C_{k, 1}, \ldots, C_{k, n}$ with

$$
C=C_{k, 0} \cup C_{k, 1} \cup \ldots \cup C_{k, n}
$$

such that

$$
\begin{aligned}
& C_{k, 0} \in \mathcal{K}(X) \cup\{\varnothing\}, \\
& C_{k, 0} \subseteq X \backslash \bigcup_{i=1}^{n} \overline{B\left(x_{i}, r_{n}\right)}, \\
& \text { for all } i=1, \ldots, n, \text { we have } C_{k, i} \in \Lambda_{t_{k, i},}, \\
& \text { for all } i=1, \ldots, n, \text { we have } C_{k, i} \subseteq B\left(x_{i}, 5 r_{n}\right) .
\end{aligned}
$$

Since $C_{k, i} \in \Lambda_{t_{k, i}, i}$, we have

$$
\int \frac{\log N_{e^{-s}}\left(C_{k, i}\right)}{s} d \Pi_{t_{k, i}}(s)>\Delta_{\Pi}\left(x_{i}, r_{n}\right)-u .
$$


This implies that for all $i=1, \ldots, n$, we have

$$
\begin{aligned}
& \overline{\operatorname{dim}}_{\Pi, B}\left(C \cap \overline{B\left(x_{i}, 5 r_{n}\right)}\right)=\limsup _{t} \int \frac{\log N_{e^{-s}}\left(C \cap \overline{B\left(x_{i}, 5 r_{n}\right)}\right)}{s} d \Pi_{t}(s) \\
& \left.\geq \limsup _{t} \int \frac{\log N_{e^{-s}}\left(C_{k, i}\right)}{s} d \Pi_{t}(s) \quad\left[\text { since } C \cap \overline{B\left(x_{i}, 5 r_{n}\right)}\right) \supseteq C_{k, i}\right] \\
& \geq \limsup _{k} \int \frac{\log N_{e^{-s}}\left(C_{k, i}\right)}{s} d \Pi_{t_{k, i}}(s) \\
& \geq \Delta_{\Pi}\left(x_{i}, r_{n}\right)-u .
\end{aligned}
$$

We conclude immediately from (7.27) that $C \notin M_{u}$. This completes the proof of Claim 4.

Combining Claim 2, Claim 3 and Claim 4, we now conclude that $M_{u}$ is meagre.

(2) This statement follows from (1) since $T=\bigcap_{n} T_{n}$.

Proposition 7.5. Let $X$ be a metric space and let $\Pi=\left(\Pi_{t}\right)_{t \geq t_{0}}$ be an averaging system. Let $\left(x_{n}\right)_{n}$ be a sequence of points from $X$, and let $\left(r_{n}\right)_{n}$ be sequence of positive real numbers. Write

$$
\begin{gathered}
T=\{C \in \mathcal{K}(X) \mid \text { for all } n \in \mathbf{N} \text { and for all } i=1, \ldots, n, \text { we have } \\
\left.\qquad \overline{\operatorname{dim}}_{\Pi, B}\left(C \cap \overline{B\left(x_{i}, 5 r_{n}\right)}\right) \geq \Delta_{\Pi}\left(x_{i}, r_{n}\right)\right\},
\end{gathered}
$$

and

$$
S=\left\{C \in \mathcal{K}(X) \mid \overline{\operatorname{dim}}_{\Pi, B}(C) \geq \sup _{x \in C} \Delta_{\Pi}(x)\right\},
$$

If $\left(x_{n}\right)_{n}$ is dense in $X$ and $r_{n} \rightarrow 0$, then $T \subseteq S$.

Proof. Let $C \in T$ and $\varepsilon>0$. First choose $x_{0} \in C$ such that

$$
\Delta_{\Pi}\left(x_{0}\right) \geq \sup _{x \in C} \Delta_{\Pi}(x)-\varepsilon \text {. }
$$

Next, choose $r_{0}>0$ such that

$$
\Delta_{\Pi}\left(x_{0}, r_{0}\right) \geq \Delta_{\Pi}\left(x_{0}\right)-\varepsilon
$$

Finally, since the sequence $\left(x_{n}\right)_{n}$ is dense in $X$, we can choose $i_{0}$ such that $x_{i_{0}} \in$ $B\left(x_{0}, \frac{r_{0}}{2}\right)$, and since $r_{n} \rightarrow 0$, we can choose $n_{0}$ such that $i_{0} \leq n_{0}$ and $r_{n_{0}} \leq \frac{r_{0}}{2}$. Since $i_{0} \leq n_{0}$ and $C \in T$, we have

$$
\overline{\operatorname{dim}}_{\Pi, B}(C) \geq \overline{\operatorname{dim}}_{\Pi, B}\left(C \cap B\left(x_{i_{0}}, 5 r_{n_{0}}\right)\right) \geq \Delta_{\Pi}\left(x_{i_{0}}, r_{n_{0}}\right) .
$$

Also, since $B\left(x_{i_{0}}, r_{n_{0}}\right) \subseteq B\left(x_{0}, \frac{r_{0}}{2}+r_{n_{0}}\right) \subseteq B\left(x_{0}, \frac{r_{0}}{2}+\frac{r_{0}}{2}\right)=B\left(x_{0}, r_{0}\right)$, it follows from Lemma 7.1 that

$$
\Delta_{\Pi}\left(x_{i_{0}}, r_{n_{0}}\right) \geq \Delta_{\Pi}\left(x_{0}, r_{0}\right) .
$$

Combining (7.28)-(7.31) yields

$$
\overline{\operatorname{dim}}_{\Pi, B}(C) \geq \Delta_{\Pi}\left(x_{i_{0}}, r_{n_{0}}\right) \geq \Delta_{\Pi}\left(x_{0}, r_{0}\right) \geq \Delta_{\Pi}\left(x_{0}\right)-\varepsilon \geq \sup _{x \in C} \Delta_{\Pi}(x)-2 \varepsilon .
$$

Finally, letting $\varepsilon \searrow 0$ gives the desired result.

We can now prove Theorem 2.2.(2).

Proof of Theorem 2.2.(2). Let the sets $S$ and $T$ be defined as in the statement of Proposition 7.5. It follows from Proposition 7.4 that $T$ is co-meagre and since $X$ is separable, it follows from Proposition 7.5 that $T \subseteq S$. We conclude from this that $S$ is co-meagre. This clearly implies the statement in the theorem. 


\section{References}

[AAOPRR] Adam-Day, D.. C. Ashrroft, L. Olsen, N. Pinzani, A. Rizzoli, and J. Rowe: On the average box dimensions of graphs of typical continuous functions. - Acta Math. Hungar. (to appear).

[Fa] Falconer, K. J.: Fractal geometry - John Wiley \& Sons, Ltd., Chichester, 1989.

[Gr] GRUBer, P.: Dimension and structure of typical compact sets, continua and curves. Monatsh. Math. 108, 1989, 149-164.

[Ha] Hardy, G. H.: Divergent series. - Oxford, 1949.

[HaYa] HARE, K., and S. YAZDANI: Quasi self-similarity and multifractal analysis of Cantor measures. - Real Anal. Exchange 27, 2001/02, 287-307.

[Ja] JACOB, M.: Über die Äquivalenz der Cesaroschen und der Hölderschen Mittel für Integrale bei gleicher reeller Ordnung $k>0$. - Math. Z. 26, 1927, 672-682.

[MyRu] Myjak, J., and R. RudNicki: Box and packing dimensions of typical compact sets. Monatsh. Math. 131, 2000, 223-226.

[O'Ne] O'NeIL, T.: The multifractal spectrum of quasi self-similar measures. - J. Math. Anal. Appl. 211, 1997, 233-257.

[Ox] Oxтовy, J.: Measure and category. A survey of the analogies between topological and measure spaces. - Grad. Texts in Math. 2, Springer-Verlag, New York-Berlin, 1971.

[Ro] Rouyer, J.: Generic properties of compact metric spaces. - Topology Appl. 158, 2011, $2140-2147$.

[St] Stroock, D.: Essentials of integration theory for analysis. - Springer Verlag, 2011.

Received 9 May 2018 • Accepted 24 July 2018 